\title{
Optimal health investment and preference structure
}

\author{
Théophile T. Azomahou ${ }^{1,2,3} \cdot$ Bity Diene $^{3}$. \\ Mbaye Diene $^{4}$. Luc Soete ${ }^{1,2}$
}

Received: 13 February 2013 / Accepted: 4 July 2015 / Published online: 12 August 2015

(C) The Author(s) 2015. This article is published with open access at Springerlink.com

\begin{abstract}
This paper develops a general equilibrium framework to study the role of preference structure (additive, multiplicative and a convex combination of the two) in connecting consumption, health investment, stock of health and capital, and their effects on the wage rate and on productivity. We show that the elasticities of health production, health investment and health cost determine jointly how health influences the wage rate. We examine the steady state and the equilibrium dynamics of the model. In the case of additive preferences, the existence of equilibrium and the stability of the dynamic system require that the ratio of the elasticities of the cost of health and health investment is greater than the elasticity of the production function of health. Health stock can have either positive or negative effects on wages via a mechanism of reservation wages. When preferences are multiplicative, the condition of the existence
\end{abstract}

$凶$ Théophile T. Azomahou

azomahou@merit.unu.edu

Bity Diene

bity.diene@udamail.fr

Mbaye Diene

mdiene@cres-sn.org

Luc Soete

1.soete@maastrichtuniversity.nl

1 United Nations University (UNU-MERIT), Boschstraat 24, 6211 AX Maastricht, The Netherlands

2 Department of Economics, Maastricht University, P.O. Box 616, 6200 MD Maastricht, The Netherlands

3 University of Auvergne, Centre d'Etude et de Recherches sur le Développement International (CERDI), 65 Boulevard François Mitterrand, Clermont-Ferrand Cedex 1, France

4 Consortium pour la Recherche Economique et Sociale (CRES), University Cheikh-Anta-Diop, Rue 10 Prolongée, Cité Iba Ndiaye Diadji No. 1-2, Dakar, Senegal 
of equilibrium and the stability of the dynamic system reverse, and the effect of health stock on wages is always positive. Longevity is a decreasing convex-concave function of the elasticity of inter-temporal substitution of health. We also compare the relative behavior of opportunity costs of health under preference structure.

Keywords Consumption · Health investment · Wage · Longevity ·

Opportunity costs

JEL Classification $\mathrm{C} 61 \cdot \mathrm{C} 62 \cdot \mathrm{I} 15 \cdot \mathrm{E} 21$

\section{Introduction}

There is a widely established consensus on the causal relation between health and longevity in the sense that better health conditions extend longevity. Some studies were even interested in the challenges of studying the conditions of a long healthy life, for a long life will often go hand in hand with a decline in health (Kirkwood 2008). This consensus crumbles when it comes to the link between health and productivity or health and economic growth. This relationship has been the subject of heated debate since the publication of the study by Acemoglu and Johnson (2007), where the authors casted serious doubts on the fact that better health would lead to faster growth per capita. Acemoglu and Johnson (2007) underlined that empirical studies that showed a sizable positive effect of health on individual productivity have not resolved the question of whether health differences are the cause of observed large differences in income since these studies do not incorporate general equilibrium effects. The most important general equilibrium effect comes from diminishing returns of work per effective unit. This is, for example, the case when physical capital is supplied inelastically. Indeed, in the presence of diminishing returns, estimations based on microdata overestimate the benefits of aggregated productivity due to improved health, especially when improving health comes with increased population. Acemoglu and Johnson (2007) showed that the increase in life expectancy associated with the increase in population may have a negative effect on income per capita for the working age population. This result is confirmed in particular for countries that have experienced high life expectancy, leading to a kind of puzzle.

Following the study of Acemoglu and Johnson (2007), other authors such as Ashraf et al. (2008) raised the same question. ${ }^{1}$ These authors used a simulation-based model that incorporates both micro- and macrocomponents and takes into account the direct effect of health on worker productivity. Contrary to the popular belief, they found a very moderate effect of improving health on income per capita. Ashraf et al. (2008) concluded that the rationale for health policies should then rely on humanitarian reasons rather than economic ones. The specific econometric questions underlying the debate are very well explained in Strittmater and Sunde (2013). ${ }^{2}$

\footnotetext{
1 See also Weil (2007, 2014).

2 See Acemoglu and Johnson (2014) and Bloom et al. (2014) to follow up the debate.
} 
This paper develops a theoretical framework to contribute to the debate by elaborating in depth on the structure of preferences. How would improving health status affect consumption, wage income and productivity? Depending on the answer, the impact on growth could be sizeable or small. This study aims at providing a theoretical answer to this question by showing that the adopted preferences do matter. To the best of our knowledge, the existing literature has not yet investigated the role of preferences in this regard.

Indeed, from the AIDS empirical literature (e.g., Bloom and Mahal 1997a, b; Cuddington and Hancok 1994), we know that consumption and health are interconnected, but we cannot make conclusions about the net effect of health investment. ${ }^{3}$ Indeed, it seems analytically difficult to slice on the net effect of a high health deterioration rate and low health productivity on health investment as not all diseases have the same effects. From a theoretical perspective, in the Grossman's (1972) standard model, health is considered as capital stock that increases with investment. Agents' preferences are separable in health and ordinary consumption. As a result, the returns from these two goods are independent. However, evidences stressed the fact that ordinary consumption is also crucial for health. Moreover, one of the main economic implications of health shock is a probable and significant distortion in savings behavior. Chakraborty (2004) considered the problem of public investment in health within the framework of overlapping generation models. The author showed that in poor countries where life expectancy is low, individuals are more likely to discount the future and thus are less inclined to save. Cuddington and Hancok (1994) also stated that health expenditure induces a decrease in savings at the expense of capital accumulation. However, this is questionable due to the fact that health expenditure is harmful to consumption. Therefore, there is an overriding issue as to how to deal with savings in the context of health depreciation.

Our study contributes to the literature in several aspects. Firstly, we adopt a more general setup by considering both separable and non-separable preferences (additive, multiplicative and a convex combination of the two) in consumption and health, meaning in the latter case consumption is also crucial for health. Second, we investigate the effects of health status on the subsequent life cycle, in particular on productivity, wage income and consumption in a general equilibrium setting. In order to have a better picture of the life cycle aspect of the issue, we also include a final good sector where productivity depends on the health stock. Thirdly, we characterize the analytical solutions of the optimization problem to study the equilibrium dynamics.

We find that the picture is quite sophisticated, depending on the assumed preferences. For simplification, we consider in a first step, lifetime of individuals as infinite. However, this facet of our approach is closely related to the framework of Grossman (1972) and Ehrlich and Chuma (1990) that health capital is still a determinant of lifetime utility. Relying on this setting, we connect three factors: health production, health investment and health costs. We show that the elasticities of these three variables determine jointly how health affects labor and hence productivity. The framework allows us to relate the evolution of wage rate with respect to health status, the transmission

\footnotetext{
3 See also Corrigan et al. (2005) and McDonald and Roberts (2006).
} 
channel between wage rate and health being labor productivity. It also enables us to study longevity as well as the opportunity cost of health investment.

The additive and multiplicative nature of preferences should be related to exogeneity versus endogeneity of health in marginal changes of welfare with respect to consumption. Relying on Grossman (1972), several empirical studies (Grossman and Benham 1974; Lee 1982; Haveman et al. 1994; Cai 2009) showed that taking into account the endogeneity of health significantly changes the results. ${ }^{4}$ These studies estimated the simultaneous effects of health and wages in a structural framework, but the findings remain mixed. While Grossman and Benham (1974) and Haveman et al. (1994) found that taking into account the endogeneity of health increases the estimated effect of health on wages, Lee (1982) showed that controlling for endogeneity reduces this effect. The study of Cai (2009) supports the results of Grossman and Benham (1974) and Haveman et al. (1994) showing that the exogeneity of health leads to an underestimation of the effect thereof on wages. Therefore, the structure of preferences is a key factor in modeling the effects of health on wages. In our study, additive preferences imply exogeneity of health in marginal changes of welfare relative to consumption, whereas multiplicative preferences imply endogeneity of it. Exogeneity means that the change in welfare following that of consumption is independent of the level of health.

When preferences are additively separable, the existence of equilibrium and the stability of the dynamic system require that the ratio of the elasticities of the cost of health and health investment is greater than the elasticity of the production function of health. The stock of health can have either positive or negative effects on the wage rate. This finding provides further clarification on the relationship between reservation wages and health. To our knowledge, few studies established this link (Gordon and Blinder 1980; Brown et al. 2009). Most studies have focused on how reservation wages influence the length of employment (Lancaster and Chesher 1983; Blackaby et al. 2007). However, there are reasons to believe that health is a determinant of reservation wages. Indeed, unhealthy people are entitled to higher reservation wages due to the disutility of labor that stems from their health status. We show that if the reservation wage is less than the market wage, the effect of health on wages is positive. If the reservation wage is higher than the market wage, the effect of health on wages becomes negative. This finding provides a general theoretical support to the empirical evidence of Gordon and Blinder (1980). Indeed, as these authors, we obtain that health has a positive effect on the reservation wage. However, while the results of Gordon and Blinder (1980) suggest that the effect of health on the market wage is negative, we theoretically show that this effect can be positive or negative depending on whether the reservation wage is less or higher than the market wage.

When the preferences are multiplicative, the condition reverses-the ratio of the elasticities of the cost of health and health investment should be lower than the elasticity of the production function of health. Moreover, the effect of health stock on wage rate is always positive. In the case of convex combination of additive and multiplicative preferences, the equilibrium dynamic of the stock of health and investment

\footnotetext{
${ }^{4}$ Endogeneity of health may follow from unobserved factors such as preferences that affect both health and wages. It can also be a result of reverse causality running from wages to health.
} 
in health is obtained as a function of time for which we observe an exponential decay. However, the solution of the dynamic system is not analytically tractable. The structure of preferences determines the shape and existence of longevity with respect to the elasticity of substitution of health. Lastly, we establish the conditions for comparing the opportunity cost of health under preference structures.

In this paper, we abstract away from the pure literature on infectious diseases and their modes of transmission (see e.g., Boucekkine and Laffargue 2010; Goenka and Liu 2012; Goenka et al. 2014). It would have been interesting to incorporate this issue in our dynamic setting to assess the impact of health on variables such as capital accumulation, consumption and wages. Here, we do not make any specific assumptions on the nature of diseases (infectious or not) as well as on their transmission process. Incorporating these mechanisms in our model will pose additional difficulties that we leave for future research while focusing on the equally difficult issue of preference structure.

The remainder of the paper proceeds as follows. Section 2 describes the general framework including optimality conditions. In Sect. 3, we study the model with separable additive preferences, where that health and consumption enter additively into the utility function. Section 4 is devoted to the model with a multiplicative non-separable preferences which allows for an interaction between health and consumption. Section 5 considers a framework with a convex combination of the additive and the multiplicative preferences. The penultimate Sect. 6 studies the opportunity costs of investment in health and derives some policy implications. The last section concludes the study. Proofs of propositions are relegated to the "Appendix."

\section{Motivational framework}

This section introduces a set of generalities of the framework that will be used subsequently. This includes functional hypotheses and optimality conditions that are required for equilibrium solutions as well separability issues regarding the preferences. The model is based on infinitely lived consumers where agent's welfare is composed of utility derived from consumption goods and health.

\subsection{Setting up and assumptions}

We assume the following:

Assumption 1 The instantaneous utility function at time $z, U(C(z)): \mathbb{R}_{+} \rightarrow \mathbb{R}_{+}$is $C^{\infty}$ with $U_{C}>0, U_{C}^{\prime}<0$ and $\lim _{C(z) \rightarrow 0} U_{C}=\infty$, where $C(z)$ denotes consumption at time $z$ and subscript means derivative with respect to concerned argument hereafter.

Assumption 2 The healthy time function (or amount of healthy time) $\varphi(M(z))$ : $\mathbb{R}_{+} \rightarrow \mathbb{R}_{+}$is $C^{\infty}$ with $\varphi_{M}>0, \varphi_{M}^{\prime}<0, \lim _{M(z) \rightarrow 0} \varphi_{M}<\infty$ and $\lim _{M(z) \rightarrow \infty} \varphi_{M}=$ 0 , where $M(z)$ denotes the stock of health capital.

Assumption 3 The health production function $\psi(m(z)): \mathbb{R}_{+} \rightarrow \mathbb{R}_{+}$is $C^{\infty}$ with $\psi_{m}>0, \psi_{m}^{\prime}<0, \lim _{m(z) \rightarrow 0} \psi_{m}<\infty$ and $\lim _{m(z) \rightarrow \infty} \psi_{m}=0$, where $m(z)$ denotes the health investment. 
Assumption 4 The production function $F(K(z), L(z)): \mathbb{R}_{+}^{2} \rightarrow \mathbb{R}_{+}$is $C^{\infty}$. Moreover,

(i) $F_{1}>0, F_{11}<0, F_{2}>0, F_{22}<0, F_{12}=F_{21}>0$ and $F_{11} F_{22}-F_{12} F_{21}>0$ where the place of the subscripts $\{1,2\}$ refers to the derivatives of the function with respect to the first and second arguments, namely $K$ and $L$.

(ii) $\lim _{K(z) \rightarrow 0} F_{1}=\infty$ and $\lim _{K(z) \rightarrow \infty} F_{1}=0$

(iii) $F(0, L(z))=F(K(z), 0)=0$.

Assumptions 1-4 are optimality conditions. They guarantee convexities of the optimization problem. The specific functional forms that will be used subsequently fulfill these postulates. Consistent with Ehrlich and Chuma (1990), we consider that the stock of health capital can be maintained or increased through purposive investments $m(z)$. However, health is submitted to a natural biological deterioration at the rate $\delta_{M}$. Thus, in contrary to Ehrlich and Chuma (1990), we assume a constant rate of health depreciation. However, the greater the health that one intends to maintain in later years, the earlier one must initiate significant investments in counteracting the depreciation of health. Let $\rho$ denote the time preference or discount rate. Individuals maximize lifetime utility, subject to the state variables. The general framework is stated as:

$$
\max \int_{0}^{\infty} \mathscr{V}\{S[U(C(z)), \varphi(M(z))], N[U(C(z)), \varphi(M(z))]\} \mathrm{e}^{-\rho z} \mathrm{~d} z
$$

subject to the law of motion of non-human assets and health:

$$
\begin{aligned}
\dot{A}(z) & =r(z) A(z)+w(z) \varphi(M(z))-C(z)-h(m(z)) \\
\dot{M}(z) & =\psi(m(z))-\delta_{M} M(z)
\end{aligned}
$$

where $r(z)$ and $w(z)$ are the interest and wage rate, respectively, and $h(m(z))$ denotes the cost of investment in health. The functions $S[U(C(z)), \varphi(M(z))]$ and $N[U(C(z)), \varphi(M(z))]$ denote the additive and non-additive components of the felicity $\mathscr{V}(S, N)$, respectively. Subsequently, three different versions of $\mathscr{V}(S, N)$, namely separable, multiplicative and a convex combination of the two, will be studied. In all these cases, the utility function $U(C(z))$ and health function $\varphi(M(z))$ will be taken as the constant relative risk aversion (CRRA) representation. We will also consider, in "Appendix 3," examples of alternative preferences for $U(C(z))$ and $\varphi(M(z))$, namely the logarithmic and the quadratic.

From the production side, we consider a representative firm with Cobb-Douglas technology for the function $F$ with the refinement that productivity depends on health deep parameters. Moreover, we assume productivity in this sector as a function of the health stock. ${ }^{5}$ This leads to $Y(z)=B(M(z)) F(K, L)$ where, $Y(z), K(z)$ and $L(z)$ are, respectively, the output, capital and labor, while $B(M(z))$ is the productivity as a function of the health stock $M$. Let the effective labor supply be $\mathscr{N}(z)=\gamma(M) L(z)$. The fraction $\gamma(M)$ shall depend on the level of health, $L(z)$ being the total labor

\footnotetext{
5 We thank two anonymous referees for suggesting the incorporation of productivity into the final sector as a function of the health stock and their suggestion to study the effect of health not only on felicity but also on labor supply and wage income.
} 
input and $\gamma(M)$ is an increasing function. As productivity depends on the stock of health, $B(M(z))=a(z) \gamma(M)^{1-\epsilon}$ with $0<\epsilon<1$ and $a(z)$ represents the technical progress which corresponds also to the global productivity. For the limit conditions of $B(M)$, the minimum level of health is assumed $M=M_{\min } \geq 0$. Furthermore, $B\left(M_{\min }\right)=a(z)$ if one assumes that $\gamma\left(M_{\min }\right)=1$. We also assume that $B(\infty)=$ $M_{\max }$ (which implies that the productivity cannot increase indefinitely) and $B_{M M} \leq 0$. Let us denote $\hat{k}=\frac{K}{L}$ the capital-labor ratio. Then, the output per labor is given by $f(\hat{k})=\frac{Y(z)}{L(z)}=B(M(z)) \hat{k}(z)^{\epsilon}$.

\subsection{Optimality}

Individuals maximize lifetime utility as stated in Eq. (1), subject to the state variables $\dot{A}(z)$ and $\dot{M}(z)$ in Eqs. (2) and (3), respectively. The Hamiltonian of this optimal control problem is given by:

$$
\begin{aligned}
H\left(C, m, M, A, \lambda_{A}, \lambda_{M}\right)= & \mathscr{V}\{S[U(C(z)), \varphi(M(z))], N[U(C(z)), \varphi(M(z))]\} \mathrm{e}^{-\rho z} \\
& +\lambda_{A}[r(z) A(z)+w(z) \varphi(M(z))-C(z)-h(m(z))] \mathrm{e}^{-\rho z} \\
& +\lambda_{M}\left[\psi(m(z))-\delta_{M} M(z)\right] \mathrm{e}^{-\rho z}
\end{aligned}
$$

The optimality conditions (where, to ease notations, the argument $z$ is removed when it is not necessary) associated with this problem are given as:

$$
\begin{aligned}
\frac{\partial H}{\partial C(z)} & =\mathrm{e}^{-\rho z}\left(-\lambda_{A}+U_{C} V_{N} N_{U}+U_{C} S_{U} V_{S}\right)=0 \\
\frac{\partial H}{\partial m(z)} & =-\mathrm{e}^{-\rho z} \lambda_{A} h_{m}+\mathrm{e}^{-\rho z} \lambda_{M} \psi_{m}=0 \\
\frac{\partial H}{\partial M(z)} & =\mathrm{e}^{-\rho z}\left(-\delta_{M} \lambda_{M}+\varphi_{M}\left(w \lambda_{M}+N_{\varphi} V_{N}+S_{\varphi} V_{S}\right)\right) \\
& =\mathrm{e}^{-\rho z}\left(\rho \lambda_{M}-\dot{\lambda}_{M}\right) \\
\frac{\partial H}{\partial A(z)} & =\mathrm{e}^{-\rho z}\left(r \lambda_{A}\right)=\mathrm{e}^{-\rho z}\left(\rho \lambda_{A}-\dot{\lambda}_{A}\right)
\end{aligned}
$$

with the associated transversality conditions:

$$
\begin{gathered}
\lim _{z \rightarrow \infty} \lambda_{A}(z) \mathrm{e}^{-\rho z} A(z)=0 \\
\lim _{z \rightarrow \infty} \lambda_{M}(z) \mathrm{e}^{-\rho z} M(z)=0
\end{gathered}
$$

The functions $\lambda_{A}(z)$ and $\lambda_{M}(z)$ are the costates, and subscripts indicate the first derivatives of functions w.r.t. mentioned arguments. The general dynamic system is given by:

$$
\frac{\dot{C}(z)}{C(z)}=-\frac{(r-\rho)\left(V_{N} N_{U}+S_{U} V_{S}\right)-\Psi_{1} \dot{M}(z) \varphi_{M}}{\Psi_{2}} \frac{U_{C}}{C(z)}
$$




$$
\begin{aligned}
\frac{\dot{m}(z)}{m(z)} & =-\frac{\Phi}{U_{C}\left(V_{N} N_{\varphi}+S_{U} V_{S}\right)\left(\psi_{m} h_{m}^{\prime}-h_{m} \psi_{m}^{\prime}\right)} \frac{\psi_{m}}{m(z)} \\
\frac{\dot{M}(z)}{M(z)} & =\frac{\psi(m(z))}{M}-\delta_{M} \\
\frac{\dot{k}}{k} & =B(M) \hat{k}^{\epsilon-1}-\frac{\hat{C}}{k}-\frac{\hat{m}}{k}-\delta
\end{aligned}
$$

where $\delta \geq 0$ is the capital depreciation rate and $w$ is the wage rate, and:

$$
\begin{aligned}
\Psi_{1}= & V_{S} S_{U \varphi}+V_{N} N_{U \varphi}+N_{\varphi}\left(V_{\varphi \varphi} N_{U}+S_{U} V_{S N}\right)+S_{\varphi}\left(N_{\varphi} V_{S N}+S_{U} V_{S S}\right) \\
\Psi_{2}= & U_{C}^{\prime}\left(V_{N} N_{U}+S_{U} V_{S}\right) \\
& +U_{C}^{2}\left(V_{\varphi \varphi} N_{U}^{2}+2 S_{U} N_{U} V_{S N}+V_{S} S_{U U}+V_{N} N_{U U}+S_{U}^{2} V_{S S}\right) \\
\Phi= & U_{C}\left(V_{N} N_{\varphi}+S_{U} V_{S}\right)\left[w \varphi_{M} \psi_{m}-\left(\delta_{M}+r\right) h_{m}\right]+\varphi_{M} \psi_{m}\left(N_{\varphi} V_{N}+S_{\varphi} V_{S}\right)
\end{aligned}
$$

Our objective consists in finding the optimal trajectories of the model key variables: consumption, investment in health, health stock and capital. Given Assumptions 1-4, our optimization program allows to get these optimal variables. It is worth noticing that it would have been interesting to incorporate infectious diseases and their modes of transmission in our dynamic setting to assess the impact of health on variables such as capital accumulation, consumption and wages. Indeed, it is interesting to know how health deterioration may affect the existence of solutions to the maximization of welfare. As well documented in Goenka and Liu (2012) and Goenka et al. (2014), if this damage was done by infectious diseases, then a problem of non-convexity arises and one needs to check existence conditions for optimal solutions. ${ }^{6}$

Goenka and Liu (2012) and Goenka et al. (2014) discussed the optimal investment in health, in the light of the interaction between the transmission of diseases and the economy. If diseases affect the labor market, health investment choices also affect the transmission of diseases. Health expenditures lead to the accumulation of health capital and thus reduce the spread of diseases and improve convalescence and recovery from illness. However, the non-convexity of the dynamics of infection implies that one should be careful in implementing the optimal control techniques. Indeed, to characterize optimal solutions, the first-order conditions (and the transversality conditions) of the Hamiltonian may be necessary but not sufficient. Then, there may be jump issues of state and co-state variables within the feasible set, whereas the existence of optimal solutions relies on compactness of the set and absolute continuity of the state variables. In this study, as stated earlier, we do not make specific assumptions on the nature of diseases (infectious or not) as well as their transmission mechanism. Incorporating these mechanisms in our model will imply extra difficulties that we leave for future research while focussing on the equally difficult separability issue in preference structure.

\footnotetext{
6 We are very much grateful to a referee who pointed out to clarify the potential non-convexity issues in the study. Indeed, Goenka and Liu (2012) and Goenka et al. (2014) showed that given the internal propagation mechanism of infectious diseases, there are non-convexities in the transmission process which make the optimization problem subtle.
} 
Some general remarks can be made before addressing the calculation of optimal trajectories. Firstly, Eq. (5a) gives the expected evolution of optimal consumption which can be disentangled into $\dot{C}(z)=C_{1}(z)+C_{2}(z)$, with $C_{1}(z)=\frac{-(r-\rho)\left(V_{N} N_{U}+S_{U} V_{S}\right) U_{C}}{\Psi_{2}}$ and $C_{2}(z)=\frac{\Psi_{1} U_{C} \dot{M}(z) \varphi_{M}}{\Psi_{2}}$. The term $C_{1}(z)$ denotes the Fisher conditions binding the slope of consumption trajectory to the difference between the time preference and interest rates. The term $C_{2}(z)$ reflects the interaction between the stock of health and consumption. When time increases, the stock of health shall move toward its minimum level, and the marginal loss of healthy time $\varphi_{M}(M(z))$ will reach its maximum, thereby reducing consumption.

Secondly, the opportunity cost of health stock (or unit cost) can be retrieved from Eq. (5b) as $\frac{\lambda_{M}}{\lambda_{A}}=g(z)$. At equilibrium, the instantaneous user cost of health stock is equal to the instantaneous marginal benefit from one-unit increase in the stock of health. The optimal health investment is determined by the intersection between the curves representing these two elements. We have not chosen an explicit specification for the cost function, but we can still derive some information from the expression of the opportunity cost of health, based on co-state variables. Thus, it can be shown from Eq. (5c) that

$$
g(M(z))\left(\delta_{M}+r-\frac{\dot{g}(z)}{g(z)}\right)=\varphi_{M}\left[w+\frac{1}{\lambda_{A}(0)}\left(N_{\varphi} V_{N}+S_{\varphi} V_{S}\right) \mathrm{e}^{(\rho-r) z}\right]
$$

The left-hand side of this equality stands for the user cost of health capital, the form of which as can be seen is comparable to that of physical capital in the theory of investment. It is also termed "marginal efficiency of capital" (Grossman 1972). The right-hand side is the effect on the utility of an increase in the stock of health. Thus, the user cost of health capital should be equal to the instantaneous marginal benefit of an increase in the stock of health. The relations (Eq. 8) can be transformed into a differential equation, say $g(t)$, which after solving, shows that the opportunity cost is proportional to:

$$
\begin{aligned}
& g(M(z)) \\
& \quad=\int_{z}^{\infty}\left[\varphi_{M}(M(x))\left(w+\frac{1}{\lambda_{A}(0)}\left(N_{\varphi} V_{N}+S_{\varphi} V_{S}\right) \mathrm{e}^{(\rho-r) x}\right)\right] \mathrm{e}^{-\left(\delta_{M}+r\right)(x-z)} \mathrm{d} x
\end{aligned}
$$

This expression gives the present value of the benefits of health stock available on the remaining life. We will use the opportunity cost later on in the penultimate section for policy purposes. In the next section, we address the case where the expression of $\mathscr{V}$ is specified to have a separable term and study the steady state and equilibrium dynamics of the model.

\section{Additively separable preferences}

The interaction between health and the ways it affects felicity has been so far investigated within additive structure of preferences (Hall and Jones 2007). As a result, health 
status and consumption are additively separable functions. The additivity of preferences implies exogeneity of health, meaning that the marginal utility of consumption is independent of health status. In terms of the optimality conditions in the previous section, exogeneity means $\frac{\partial^{2} V}{\partial \varphi \partial U}=0$ or $V_{S} S_{U \varphi}=0$. While adopting this approach in this section, let us remember that we add a final good sector to better understand the life cycle aspect of the agent's behavior. In that case, the welfare function $\mathscr{V}$ turns to take the form:

$$
\begin{aligned}
\mathscr{V}\{S[U(C(z)), \varphi(M(z))], N[U(C(z)), \varphi(M(z))]\} & =S[U(C(z)), \varphi(M(z))] \\
& =U(C(z))+\varphi(M(z))
\end{aligned}
$$

We shall also resort to the constant relative risk aversion (CRRA) felicity function, which has the following functional forms for $U(C(z))$ and $\varphi(M(z))$, and a decreasing return in health investment for function $\psi(m(z))$ :

$$
\begin{aligned}
& U(C(z))=\frac{C(z)^{1-\sigma_{1}}}{1-\sigma_{1}} \text { and } \varphi(M(z))=\frac{M(z)^{1-\sigma_{2}}}{1-\sigma_{2}} \\
& h(m(z))=\pi m(z)^{\alpha} \text { and } \psi(m(z))=b m^{\beta}
\end{aligned}
$$

with $\sigma_{1}<1, \sigma_{2}<1, b>0, \beta>0, \pi>0$ and $0<\alpha<1$. Here $\sigma_{1}$ is the inverse of elasticity of substitution between consumption at any two points in time, and $\sigma_{2}$ denotes the same for health capital. $U(C(z))$ and $\varphi(M(z))$ are strictly increasing and concave, respectively, in $C(z)$ and $M(z) . \psi(m(z))$ represents the health investments function, which is concave in $m(z)$, reflecting the assumed diminishing returns in health investment. $\pi$ is the productivity or efficiency of health investment. Increased healthcare productivity not only shifts the health production function upward, but also causes each unit of health care to have a larger contribution to health as well. Ehrlich and Chuma (1990) also assumed that consumers choose death when the stock of capital $M(z)$ is under a certain minimal level $M_{\min }$. All these functions fulfill Assumptions 1-3.

\subsection{Steady state}

The first-order conditions with respect to $C(z)$ and $A(z)$ from the Hamiltonian (4) yield the traditional Euler equations. One can see that Eqs. (7a) and (7b) turn to be, respectively:

$$
\frac{\dot{C}(z)}{C(z)}=0 \Longleftrightarrow(r-\rho) S_{U} V_{S}-\left[V_{S} S_{U \varphi}+S_{\varphi} S_{U} V_{S S}\right] \dot{M}(z) \varphi_{M}=0
$$

and

$$
\frac{\dot{m}(z)}{m(z)}=0 \Longleftrightarrow U_{C} S_{U}\left[w \varphi_{M} \psi_{m}-\left(\delta_{M}+r\right) h_{m}\right]+\varphi_{M} \psi_{m} S_{\varphi}=0
$$

Thus, Eq. (13) defines a differential equation in $M(t)$ which allows us to find the value $M^{*}(t)$ at equilibrium. The latter can then be introduced into (14) to find consumption equilibrium $C^{*}(t)$ given that the stock of health and investment are 
linked by the relation (7c). More specifically, using Eqs. (11) and (12), we obtain the demand-side system:

$$
\begin{aligned}
& \frac{\dot{C}}{C}=\frac{r-\rho}{\sigma_{1}} \\
& \frac{\dot{m}}{m}=\frac{-\varphi_{M} \psi_{m}^{2}+U_{C}\left[(r-\rho) h_{m} \psi_{m}+\left(\left(\delta_{M}+\rho\right) h_{m}-w \varphi_{M} \psi_{m}\right)\right] \psi_{m}}{U_{C} m\left(\psi_{m} h_{m}^{\prime}-h_{m}\right) \psi_{m}^{\prime}} \\
& \frac{\dot{M}}{M}=\frac{b m^{\beta}}{M}-\delta_{M}
\end{aligned}
$$

Proceeding with the final good sector, remember that $f(\hat{k})=\frac{Y(z)}{L(z)}=B(M(z)) \hat{k}(z)^{\epsilon}$. Then, the maximization of the profit function under perfect competition allows to equalize the marginal cost of each factor with its marginal benefit. Therefore,

$$
\begin{aligned}
r(z) & =\epsilon B(M(z)) \hat{k}(z)^{\epsilon-1}-\delta \\
w(z) & =f(\hat{k}(z))-\hat{k}(z) f^{\prime}(\hat{k}(z))=(1-\epsilon) B(M(z)) \hat{k}(z)^{\epsilon}
\end{aligned}
$$

Combining the demand and the supply sides, we can now characterize the equilibrium of the economy. We can write

$$
\dot{\hat{k}}(z)=B(M(z)) \hat{k}(z)^{\epsilon}-\hat{C}(z)-\hat{m}(z)-(\delta+n) \hat{k}(z)
$$

where $\hat{C}(z)$ and $\hat{m}(z)$ are, respectively, the consumption and health expenditure per labor and $n$ is the population growth rate. Therefore, the dynamics of the economy can be summarized by the following non-trivial four-dimensional system:

$$
\begin{aligned}
\frac{\dot{\hat{C}}(z)}{\hat{C}(z)} & =\frac{r(z)-\rho}{\sigma_{1}} \\
\frac{\dot{\hat{m}}(z)}{\hat{m}(z)} & =\frac{\delta_{M}+r}{\alpha-\beta}-\frac{b \beta\left(w+C^{\sigma_{1}}\right) M^{-\sigma_{2}} m^{\beta-\alpha}}{\pi \alpha(\alpha-\beta)} \\
\dot{\hat{M}}(z) & =b m^{\beta}-\delta_{M} \hat{M}(z) \\
\dot{\hat{k}}(z) & =B(M(z)) \hat{k}(z)^{\epsilon}-\hat{C}(z)-\hat{m}(z)-\delta \hat{k}(z)
\end{aligned}
$$

including $\hat{k}(0)$ and $\hat{M}(0)$ as given and in addition the transversality conditions. The steady-state values of $\hat{C}, \hat{m}, \hat{M}$ and $\hat{k}$ are obtained by equalizing $\dot{\hat{C}}, \dot{\hat{m}}, \dot{\hat{M}}, \dot{\hat{k}}$ to zero. We obtain:

$$
\begin{aligned}
& \widehat{C}=\frac{w}{1-\epsilon}\left(1-\frac{\epsilon}{r+\delta}\right)-\left(\frac{\delta_{M}}{b}\right)^{\frac{1}{\beta}} \widehat{M}^{\frac{1}{\beta}} \\
& \widehat{m}=\left(\frac{\delta_{M}}{b}\right)^{\frac{1}{\beta}} \widehat{M}^{\frac{1}{\beta}}
\end{aligned}
$$




$$
\begin{aligned}
\widehat{M}^{\sigma_{2}-1+\frac{\alpha}{\beta}} & =\frac{b\left(\frac{b}{\delta_{M}}\right)^{-\sigma_{2}} \beta}{\pi\left(\delta_{M}+r\right) \alpha}\left[w+\left(\frac{w}{1-\epsilon}\left(1-\frac{\epsilon}{r+\delta}\right)-\left(\frac{\delta_{M}}{b}\right)^{\frac{1}{\beta}} \widehat{M}^{\frac{1}{\beta}}\right)^{\sigma_{1}}\right] \\
\hat{k} & =B(M)^{\frac{1}{1-\epsilon}}\left(\frac{\delta+\rho}{\epsilon}\right)^{\frac{1}{\epsilon-1}}
\end{aligned}
$$

The following proposition characterizes the solution of the system.

Proposition 1 There is a unique solution to Eq. (20c).

Proof See "Appendix 1."

The next proposition states the comparative statics of the model as regard the effect of health stock on the wage rate. It also states the conditions of existence for the equilibrium and the stability of the dynamic system.

Proposition 2 The effect of health stock on the wage rate is positive provided that $\alpha \geq \beta\left(1-\sigma_{2}\right)$ and $\alpha>\beta$. Moreover, there exists a minimum wage rate $w_{0}$ from which the stock of health impacts positively on the wage rate.

Proof See "Appendix 1."

The condition in Proposition 2 means that the ratio of the elasticities of the cost of health and health investment is greater than the elasticity of the production function of health. In addition, for the stock of health to have a positive effect on the wage rate, it is necessary that the wage rate remains higher than a minimum level $w_{0}$. We seek for conditions under which the minimum level $w_{0}$ can be determined. Relying on Eq. (42, see "Appendix 1"), setting $\mathscr{W}_{\mathscr{N}} \geq 0$ and $\mathscr{W}_{\mathscr{D}}>0$ is equivalent to writing, respectively, $w \geq f_{1}(M)$ and $w>f_{2}(M)$ where

$$
\begin{aligned}
f_{1}(M)= & \frac{(r+\delta)(1-\epsilon)}{r+\delta-\epsilon}\left[\left(\frac{\delta_{M}}{b}\right)^{\frac{1}{\beta}} M^{\frac{1}{\beta}}\right. \\
& +\left(\frac{\left.\left.\left(\frac{b}{\delta_{M}}\right)^{\sigma_{2}}\left(\frac{\delta_{M}}{b}\right)^{-\frac{1}{\beta}} M^{\frac{-1+\alpha-\beta+\beta \sigma_{2}}{\beta} \pi\left(\delta_{M}+r\right) \alpha\left(\beta-\alpha-\beta \sigma_{2}\right)}\right)^{\frac{1}{-1+\sigma_{1}}}\right]}{b \beta \sigma_{1}}\right] \\
f_{2}(M)= & \frac{(r+\delta)(1-\epsilon)}{r+\delta-\epsilon}\left[\left(\sigma_{1} \frac{\epsilon-r-\delta}{(\epsilon-1)(r+\delta)}\right)^{\frac{1}{1-\sigma_{1}}}+\left(\frac{\delta_{M}}{b}\right)^{\frac{1}{\beta}} M^{\frac{1}{\beta}}\right]
\end{aligned}
$$

Figure 1 shows the curves $f_{1}(M)$ and $f_{2}(M)$. The dashed curve from the origin becomes a solid line from $M_{0}$, while the solid curve from $w_{0}$ becomes a dashed line from $M_{0}$. We have: 


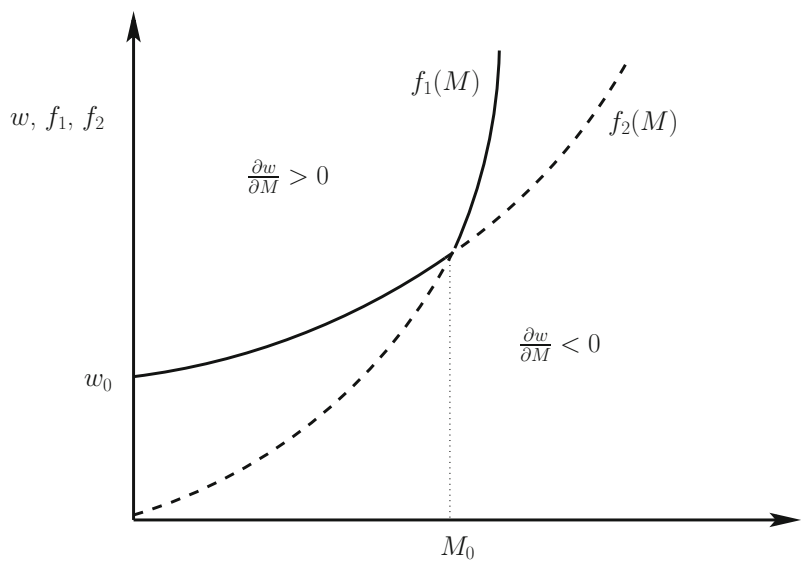

Fig. 1 Domains of wage rate

$$
\begin{aligned}
& M_{0}=\left[\frac{\frac{r+\delta-\epsilon}{(1-\epsilon)(r+\delta)}\left(\frac{b}{\delta_{M}}\right)^{\frac{1}{\beta}+\sigma_{2}} \pi\left(\delta_{M}+r\right) \alpha\left(\alpha-\beta+\beta \sigma_{2}\right)}{b \beta}\right]^{\frac{\beta}{1-\alpha+\beta-\beta \sigma_{2}}} \\
& w_{0}=\frac{(r+\delta)(1-\epsilon)}{r+\delta-\epsilon}\left[\sigma_{1} \frac{r+\delta-\epsilon}{(1-\epsilon)(r+\delta)}\right]^{\frac{1}{1-\sigma_{1}}}
\end{aligned}
$$

In fact, the influence of the stock of health on the wage rate is positive in the area bounded by the $y$-axis and the solid curve, given that the minimum ordinate is $w_{0}$. This domain is $\sup \left(f_{1}, f_{2}\right)(M)$. It is therefore possible that the stock of health has a negative effect on the wage rate and this finding is more related to the specification of the welfare function. Indeed, in the case of additive preferences, we find that $S_{U \varphi}$ and $V_{S S}$ from Eq. (13) vanish. As a result, the solution $M^{*}(z)$ is derived from Eq. (14). Relying on Eq. (7b), the expression of the wage rate is obtained as:

$$
w\left(M^{*}(z)\right)=\frac{\left(\delta_{M}+r\right) h_{m}\left[m^{*}(z)\right]}{\varphi_{M}\left[M^{*}(z)\right] \psi_{m}\left[m^{*}(z)\right]}-\frac{S_{\varphi}\left[M^{*}(z)\right]}{U_{C}\left[C^{*}(z)\right] S_{U}\left[C^{*}(z)\right]}
$$

where the variables $m^{*}(z)$ and $C^{*}(z)$ are expressed in function of $M^{*}(z)$.

In our framework, $w_{0}$ can be interpreted as the minimum of reservation wages for all possible levels of health. It is therefore a minimum level $w_{0}$ corresponding to the minimum of market wages from which the influence of health on the wages is positive. This depends on the capital factor through the interest rate $r$. Indeed, the derivative of $w_{0}$ with respect to $r$ is positive. This implies that when capital becomes more expensive (the relative price of capital increases), labor demand from firms will increase by substitution effect. These new job opportunities can lead workers to update up their reservation wages, which leads to an increase in $w_{0}$. As a result, if the reservation wage is less than the market wage, the effect of health on wages is positive. 
If the reservation wage is higher than the market wage, the effect of health on wages becomes negative.

Our finding also fills two gaps in the literature. The first is the absence of a comprehensive approach, which shows the role of health in a flexible theoretical framework, without introducing morbidity constraints or constraints related to demographic pressures of the population. Hence, our general equilibrium approach can show that the economic effects of health, measured through the wage rate (and therefore productivity), may be positive, but only under certain conditions, especially related to quality of health and costs of health investments. The second gap is the evidence of negative effects of health on the economic sphere. Acemoglu and Johnson (2007) showed empirically that the effects of health (measured by longevity) on growth of GDP per capita can be negative. We provide a theoretical basis to this finding. Intuitively, a high stock of health may well have a negative effect on the wage rate if economic growth (and hence the distribution of income) is reduced due to aging population, for example, or because of high opportunity cost of health spending which harms economic sectors. This is also possible if improved health leads to a reduction in capital per capita and thus lower levels of income per capita.

\subsection{Phase diagrams}

Let us now study the equilibrium dynamics of the system with phase diagrams. To this end, we express the variables in units of physical capital by setting: $c=\frac{C}{k}, \bar{m}=\frac{m}{k}$ and $\bar{M}=\frac{M}{k}$. It follows that the variables follow the system:

$$
\begin{aligned}
\frac{\dot{c}}{c}= & \frac{r-\rho}{\sigma_{1}}-\frac{r+\delta(1-\epsilon)}{\epsilon}+c+\bar{m} \\
\frac{\dot{m}}{m}= & \frac{\delta_{M}+r}{\alpha-\beta}-\frac{b \beta\left(w+k^{\sigma_{1}} c^{\sigma_{1}}\right) \bar{M}^{-\sigma_{2}} m^{\beta-\alpha} k^{\beta-\alpha-\sigma_{2}}}{\pi \alpha(\alpha-\beta)} \\
& -\frac{r+\delta(1-\epsilon)}{\epsilon}+c+\bar{m} \\
\frac{\dot{M}}{M}= & \frac{b \bar{m}^{\beta} k^{\beta-1}}{\bar{M}}-\delta_{M}-\frac{r+\delta(1-\epsilon)}{\epsilon}+c+\bar{m}
\end{aligned}
$$

The condition in Proposition 2 also ensures the existence and uniqueness of the steady state and guarantees the stability. Relying on the implicit behavior of $\bar{m}$ and values of parameters, we prove below that this leads to the existence and uniqueness of the solution. We are now interested in the changes in the equilibrium, when some health parameters are modified. We propose a geometrical representation by drawing the phase diagrams associated with the system (24a)-(24c). The diagrams are plotted on different planes while fixing each of the variables. Lemma 1 elaborates on the phase diagrams in Figs. 2, 3 and 4.

\section{Lemma 1 We have:}

(i) For $\bar{m}$ fixed, the curve $\dot{c}=0$ is a horizontal line and the locus $\dot{\bar{M}}=0$ is an increasing and concave function. Moreover, we have $\lim _{\bar{M} \rightarrow \infty} c=b_{2}$ for $b_{2}$ given. 
(ii) For c fixed, the curve $\dot{\bar{M}}=0$ monotonically increases and the locus $\dot{\bar{m}}=0$ is a decreasing and convex function with $\lim _{\bar{m} \rightarrow 0} \bar{M}=0$ and $\lim _{\bar{m} \rightarrow b^{-}} \bar{M}=\infty$.

(iii) For $\bar{M}$ fixed, the curve $\dot{c}=0$ is a decreasing straight line and the locus $\dot{\bar{m}}=0$ is an increasing function over a real support and it decreases monotonically from $\bar{m}_{0}$.

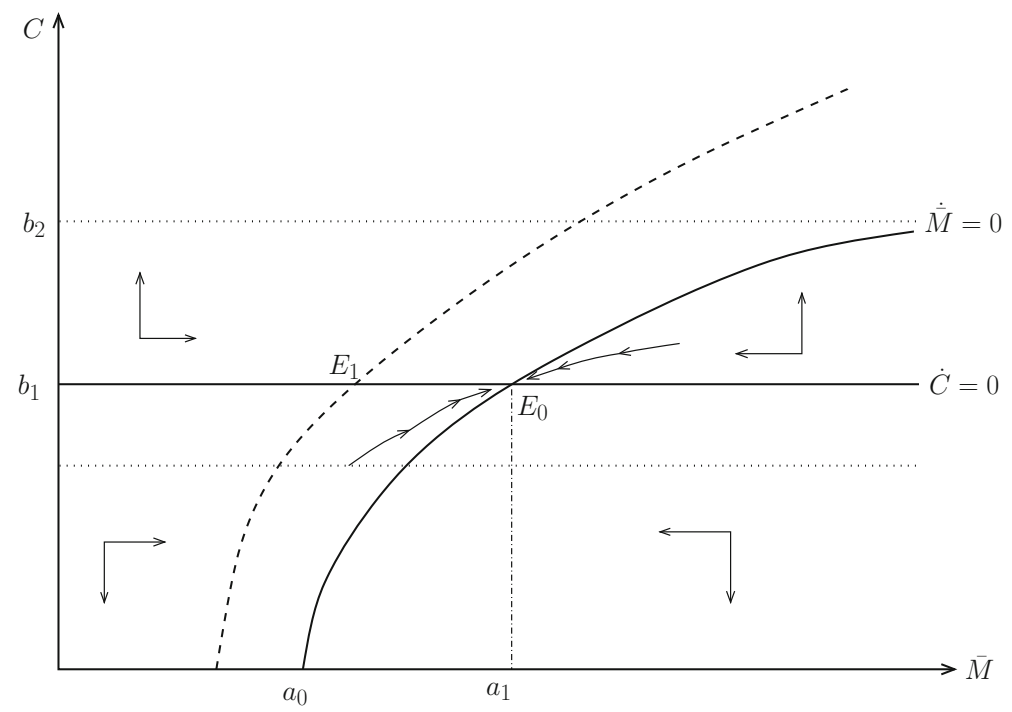

Fig. 2 Phase diagram for additive separable preferences, plane $(C, \bar{M})$

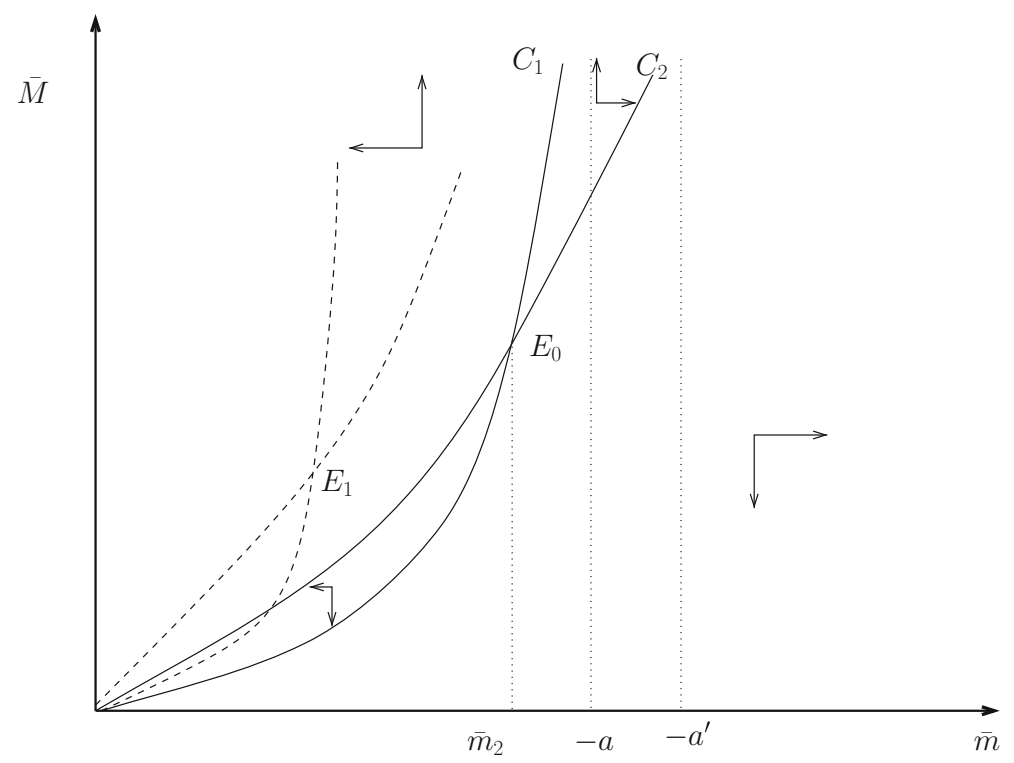

Fig. 3 Phase diagram for additive separable preferences, plane $(\bar{M}, \bar{m})$ 


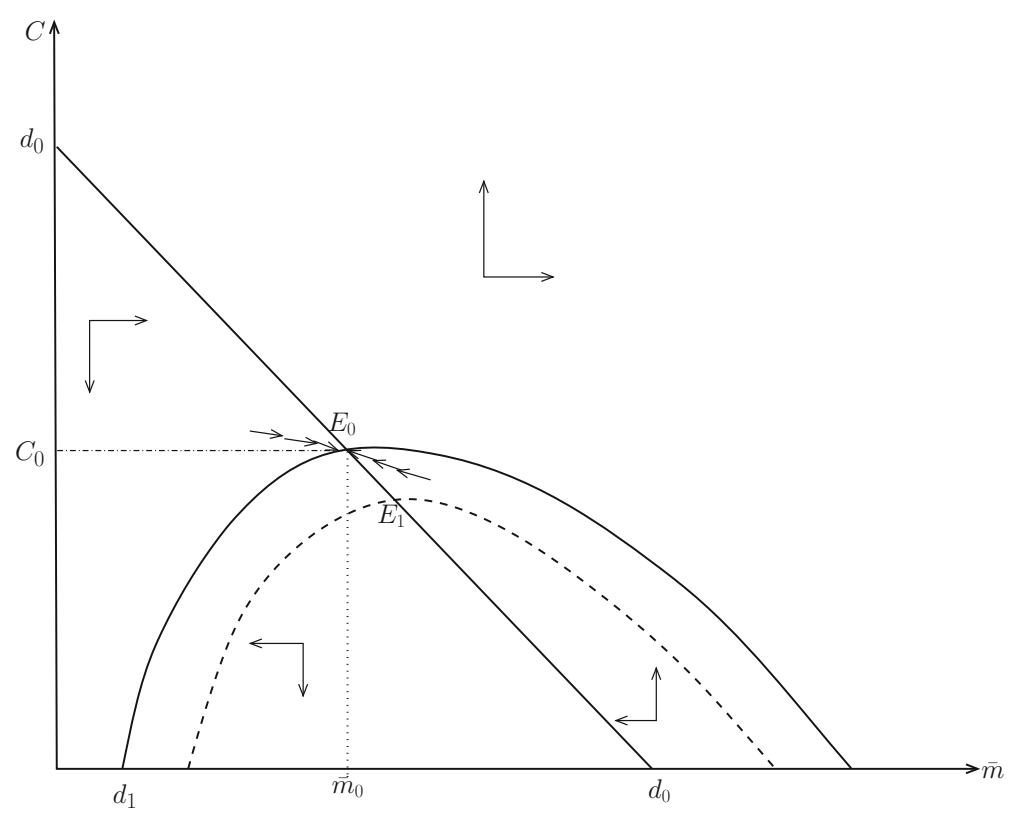

Fig. 4 Phase diagram for additive separable preferences, plane $(C, \bar{m})$

Fixing $\bar{m}=\bar{m}_{0}$ leads to the phase diagram onto the plane $(c, \bar{M})$ (see Fig. 2 ). We have two curves. The first one $(\dot{c}=0)$ is independent of the stock of health. An increase in the rate of health depreciation $\left(\delta_{M}\right)$ implies a shift of the second curve $(\dot{\bar{M}})$ toward the left. This curve is increasing with both consumption and health. The shift induces a decrease in health from the steady state $E_{0}$ to a new one, $E_{1}$, where consumption remains constant.

The second phase diagram in Fig. 3 is plotted onto the plane $(\bar{m}, \bar{M})$ by fixing $c$. The two curves are increasing with the variables $\bar{m}$ and $\bar{M}$, but the stock of health grows faster than the flow of investment. An increasing rate of health depreciation leads to a high reduction in the stock of health which is not fully compensated by the investment. So the two curves shift to the left toward the steady state $E_{1}$.

Fixing $\bar{M}$ gives the third diagram in Fig. 4 on the plane $(c, \bar{m})$. Relying on the equation $(\dot{c}=0)$, there is a linear relation between the flow of investment and consumption. The stable manifold is in the zones on the left-hand side of $E_{0}$ above the curve and the right-hand side of $E_{0}$ below the curve. Within these two zones, the trajectories converge to the steady-state values. An increase in $\delta_{M}$ generates a higher level of investment flows. Moreover, a crowding effect appears and consumption jumps backward from the first steady state $E_{0}$ to the second one, $E_{1}$.

\section{Multiplicative preferences}

In the previous section, consumption and health enter into the utility function in an additive way. As a result, the marginal utility of consumption is independent from 
health, which reflects a strong limitation as consumption is also crucial for health. The alternative model in this section seeks to account for this important aspect. Multiplicative preferences imply endogeneity of health meaning that the marginal utility of consumption depends on health status. In terms of the optimality conditions, endogeneity means $V_{N} N_{U \varphi}>0$. The welfare function $\mathscr{V}$ now takes the form:

$$
\begin{aligned}
\mathscr{V}\{S[U(C(z)), \varphi(M(z))], N[U(C(z)), \varphi(M(z))]\} & =N[U(C(z)), \varphi(M(z))] \\
& =U(C(z)) \varphi(M(z))
\end{aligned}
$$

In order to be able to consistently compare the results, we use the same CRRA felicity functional forms for $U(C(z))$ and $\varphi(M(z))$ as in Eq. (11), as well as the same health functions $h(m(z))$ and $\psi(m(z))$ as in Eq. (12). The description of final sector is the same. Given this new setup, we can study the steady state and equilibrium dynamics of the non-separable multiplicative preferences model.

\subsection{Steady state}

Here, Eqs. (7a) and (7b) turn to be (respectively):

$$
\frac{\dot{C}(z)}{C(z)}=0 \Longleftrightarrow(r-\rho) V_{N} N_{U}-\left[V_{N} N_{U \varphi}+N_{\varphi} N_{U} V_{\varphi \varphi}\right] \dot{M}(z) \varphi_{M}=0
$$

and

$$
\frac{\dot{m}(z)}{m(z)}=0 \Longleftrightarrow U_{C}\left[w \varphi_{M} \psi_{m}-\left(\delta_{M}+r\right) h_{m}\right]+\varphi_{M} \psi_{m}=0
$$

As previously stated, Eq. (26) defines a differential equation in $M(t)$ which allows us to find the value $M^{*}(t)$ at equilibrium. The latter can then be plugged into (27) to retrieve equilibrium consumption $C^{*}(t)$, thanks to relation $(7 \mathrm{c})$. The dynamics of the economy at equilibrium is driven by the following system:

$$
\begin{aligned}
\frac{\dot{\hat{C}}(z)}{\hat{C}(z)}= & \frac{(r-\rho) M(z)+\left(\delta_{M} M(z)-b m(z)^{\beta}\right)\left(\sigma_{2}-1\right)}{M(z) \sigma_{1}} \\
\frac{\dot{\hat{m}}(z)}{\hat{m}(z)}= & \frac{1}{\pi \alpha(\alpha-\beta)\left(1+\sigma_{1}\right) M(z)^{1+\sigma_{2}}}\left[\pi\left(\delta_{M}+r\right) \alpha M(z)^{1+\sigma_{2}}\left(1+\sigma_{1}\right)\right. \\
& \left.-b \beta m(z)^{\beta-\alpha}\left(w M(z)\left(1+\sigma_{1}\right)+C(z) M(z)^{\sigma_{2}}\right)\left(1+\sigma_{2}\right)\right] \\
\dot{\hat{M}}(z)= & -\delta_{M}+\frac{b m^{\beta}}{m(z)} \\
\dot{\hat{k}}_{z} & =B(M(z)) \hat{k}(z)^{\epsilon}-\hat{C}(z)-\hat{m}(z)-\delta \hat{k}(z)
\end{aligned}
$$


with $\hat{k}(0)$ and $\hat{M}(0)$ given, plus the transversality conditions. The steady-state values of $\hat{C}, \hat{m}, \hat{M}$ and $\hat{k}$ are obtained by equalizing $\dot{\hat{C}}, \dot{\hat{m}}, \dot{\hat{M}}$ and $\dot{\hat{k}}$ to zero. Doing so gives:

$$
\begin{aligned}
\widehat{C} & =\frac{w}{1-\epsilon}\left(1-\frac{\epsilon}{r+\delta}\right)-\left(\frac{\delta_{M}}{b}\right)^{\frac{1}{\beta}} \widehat{M}^{\frac{1}{\beta}} \\
\widehat{m}= & \left(\frac{\delta_{M}}{b}\right)^{\frac{1}{\beta}} \widehat{M}^{\frac{1}{\beta}} \\
\widehat{M}^{\sigma_{2}+\frac{\alpha}{\beta}-1}= & \frac{b \beta}{\pi\left(\delta_{M}+r\right) \alpha}\left(\frac{\delta_{M}}{b}\right)^{\frac{\beta-\alpha}{\beta}} \\
& {\left[w+\left(\frac{w(2(r+\delta)-\delta \epsilon)}{(r+\delta)(1-\epsilon)}-\left(\frac{\delta_{M} M}{b}\right)^{\frac{1}{\beta}}\right) \widehat{M}^{\sigma_{2}-1} \frac{\sigma_{2}-1}{\sigma_{1}-1}\right] } \\
\hat{k}= & B(M)^{\frac{1}{1-\epsilon}}\left(\frac{\delta+\rho}{\epsilon}\right)^{\frac{1}{\epsilon-1}}
\end{aligned}
$$

The following results hold:

Proposition 3 (i) The dynamic system (29a)-(29d) admits a stable solution.

(ii) The effect of health stock on wage rate is positive provided that $\beta\left(1-\sigma_{2}\right) \leq \alpha<\beta$.

Proof See "Appendix 1."

To study how the wage rate behaves in this case, one can rely on Eq. (29c). Solving the latter with respect to wage leads to:

$$
w(M)=\frac{\left(\frac{\delta_{M}}{b}\right)^{\frac{\alpha}{\beta}} M^{-1+\sigma_{2}} \pi\left(\delta_{M}+r\right) \alpha\left(M^{\frac{\alpha}{\beta}}+\frac{\delta_{M}\left(\frac{\delta_{M}}{b}\right)^{-\frac{\alpha}{\beta}}\left(\frac{\delta_{M} M}{b}\right)^{\frac{1}{\beta}} \beta\left(-1+\sigma_{2}\right)}{\pi\left(\delta_{M}+r\right) \alpha\left(-1+\sigma_{1}\right)}\right)}{\delta_{M} \beta\left(1+\frac{M^{-1+\sigma_{2}(2 r+2 \delta-\delta \epsilon)\left(-1+\sigma_{2}\right)}}{(r+\delta)(1-\epsilon)\left(-1+\sigma_{1}\right)}\right)}
$$

which is fully expressed in terms of $M$. Here, one can check $w(M)$ is an increasing function of $M$ (see "Appendix 1," proof of Proposition 3).

As we can see from Propositions 2 and 3, the conditions for the wage rate to be positively related to health stock are different. Both cases share a common condition, which is $\alpha \geq \beta\left(1-\sigma_{2}\right)$. However, whereas in the additive case one needs in addition $\alpha>\beta$, the multiplicative preference requires $\alpha<\beta$ leading to the inequality condition in Proposition 3. The latter states that the ratio of the elasticities of the cost of health and health investment must be lower than the elasticity of the production function of health. The stock of health has throughout a positive effect on the wage rate. Compared to the additive preference, we no longer have the domain of negative effect of stock of health on the wage rate which was displayed in Fig. 1. Formally, this result clearly follows from the structure of preferences. However, from an economic and empirical perspective, how could one explain this change in wage rate with respect to preference structure. 
In the case of additive preferences, health status and consumption are additively separable in the utility function, implying that the marginal utility of consumption is independent of health status. This does not fit in, for instance, with the notion that good nutrition is also important for health. Indeed, healthy eating might lead to a reduction in mortality from chronic illness, and appropriate dietary advice can prevent physical and mental deterioration and improve the quality of life. Evidence from Friis and Michaelsen (1998) supports this rationale. Therefore, consumption is also crucial for health. The case of multiplicative non-separable preference highlights the interaction between consumption and health although it is very difficult to slice on the net effect of a high health deterioration rate and low health productivity on health investment.

Another way to interpret the common relationship $\alpha \geq \beta\left(1-\sigma_{2}\right)$ between the Propositions 2 and 3 is to consider the changes in inter-temporal substitution of health with respect to health investment and the cost of that investment. Indeed, it appears that the higher the health investment (i.e., $\beta$ ), the lower the elasticity of substitution. Inter-temporal substitutability of health can become zero if the cost of investment in health becomes increasingly high. In order to improve substitutability of health stock over time, justifying a reduction in inertia of household health behavior, there must be a combination of two phenomena: a gradual decrease in the elasticity of health production and a simultaneous increase in the costs of health investment. This may seem against intuition. However, it should be noted that if households determine the level of current health stock taking into account its level in the previous period, this may affect the future marginal utility of health stock. Indeed, any increase in the level of health of the current period increases the future marginal utility of health. The effect of inter-temporal substitution between present and future stocks becomes weak if health habits are persistent. In this case, the representative household has to spend much more wealth between the current period and the future period to improve the stock of future health. And even with a low rate of health depreciation, the costs of health investments become increasingly high.

\subsection{Phase diagrams}

We now elaborate on how the health parameters affect the steady-state values, notably the health investment variable and the consequences on consumption, capital stock and savings. To this end, we study the equilibrium dynamics of this economy. Using the same variable definitions as above, the dynamic system is computed as:

$$
\begin{aligned}
\frac{\dot{c}}{c}= & \frac{-(r-\rho) M+\left(-1+\sigma_{2}\right)\left(-\delta_{M} M+b m^{\beta} k^{-\beta+1}\right)}{-\sigma_{1} M}-\frac{r+\delta(1-\epsilon)}{\epsilon}+c+\bar{m} \\
\frac{\dot{m}}{m}= & c^{\sigma_{1}} m^{1-\alpha} M^{\sigma_{2}} k^{-\sigma_{2}+\alpha-1}\left(\left(b m^{-1+\beta} M^{-\sigma_{2}} k^{\sigma_{2}-\beta+1}\right.\right. \\
& \left(\frac{c^{1-\sigma_{1}}}{-1+\sigma_{1}}+w c^{-\sigma_{1}}\left(\frac{M^{1-\sigma_{2}} k^{\sigma_{2}-1}}{-1+\sigma_{2}}\right)\right)+\pi\left(-\delta_{M}-r\right) \alpha c^{-\sigma_{1}} m^{-1+\alpha} k^{1-\alpha}
\end{aligned}
$$




$$
\begin{aligned}
& \left.\left.\left(\frac{M^{1-\sigma_{2}} k^{\sigma_{2}-1}}{-1+\sigma_{2}}\right)\right)\left(-1+\sigma_{2}\right)\right) /\left(\pi \alpha(\alpha-\beta)\left(\left(-M k^{-1}\right)\right)\right) \\
& -\frac{r+\delta(1-\epsilon)}{\epsilon}+c+\bar{m} \\
\frac{\dot{M}}{M}= & \frac{b \bar{m}^{\beta} k^{\beta+1}}{\bar{M}}-\delta_{M}-\frac{r+\delta(1-\epsilon)}{\epsilon}+c+\bar{m}
\end{aligned}
$$

Here again, we turn to the analysis of the changes in equilibrium when some health parameters are modified. Lemma 2 documents on the phase diagrams in Figs. 5, 6 and 7 which are associated with the system in Eq. (31).

\section{Lemma 2 We have:}

(i) For $\bar{m}$ fixed, the curve $\dot{c}=0$ is a horizontal line and the locus $\dot{\bar{M}}=0$ is an increasing and concave function, with $\lim _{\bar{M} \rightarrow \infty} c=a^{\prime}$ for $a^{\prime}$ given.

(ii) For c fixed, the curves $\dot{\bar{m}}=0$ and $\dot{\bar{M}}=0$ are convex and monotonically increasing with $\lim _{\bar{m} \rightarrow a} \bar{M}=\infty$ and $\lim _{\bar{m} \rightarrow a^{\prime}} \bar{M}=\infty$ for a and $a^{\prime}$ given.

(iii) For $\bar{M}$ fixed, the curve $\dot{c}=0$ is decreasing, and the locus $\dot{\bar{m}}=0$ is an increasing function up to $\bar{m}_{1}$ from which it monotonically decreases.

We fix $\bar{m}$ and get the diagram in Fig. 5 onto the plane $(c, \bar{M})$. As for the additive case, the curve $\dot{c}=0$ does not depend on the stock of health $\bar{M}$. The consumption depends though only on the flow of investment and on the rate of health depreciation. The curve $\dot{\bar{M}}=0$ is concave and increases with both variables. Starting from the steady state $E_{0}$, an increase in $\delta_{M}$ shifts upward the level of consumption, leading to the final equilibrium point $E_{1}$. The depreciation of health reduces the stock of health,

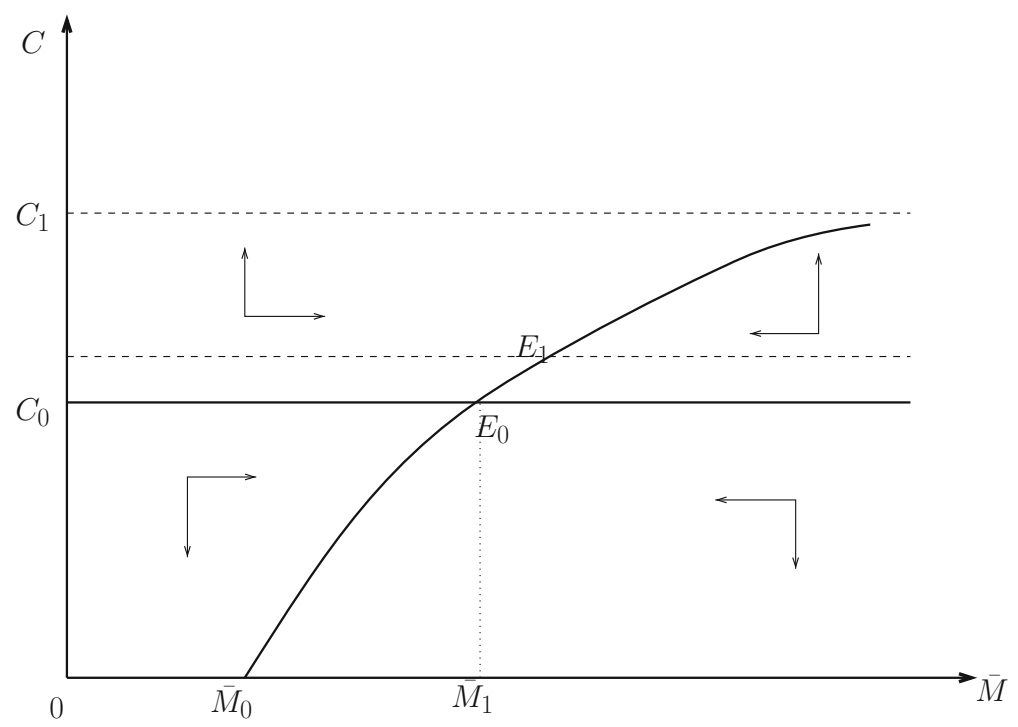

Fig. 5 Phase diagram for multiplicative non-separable preferences, plane $(C, \bar{M})$ 


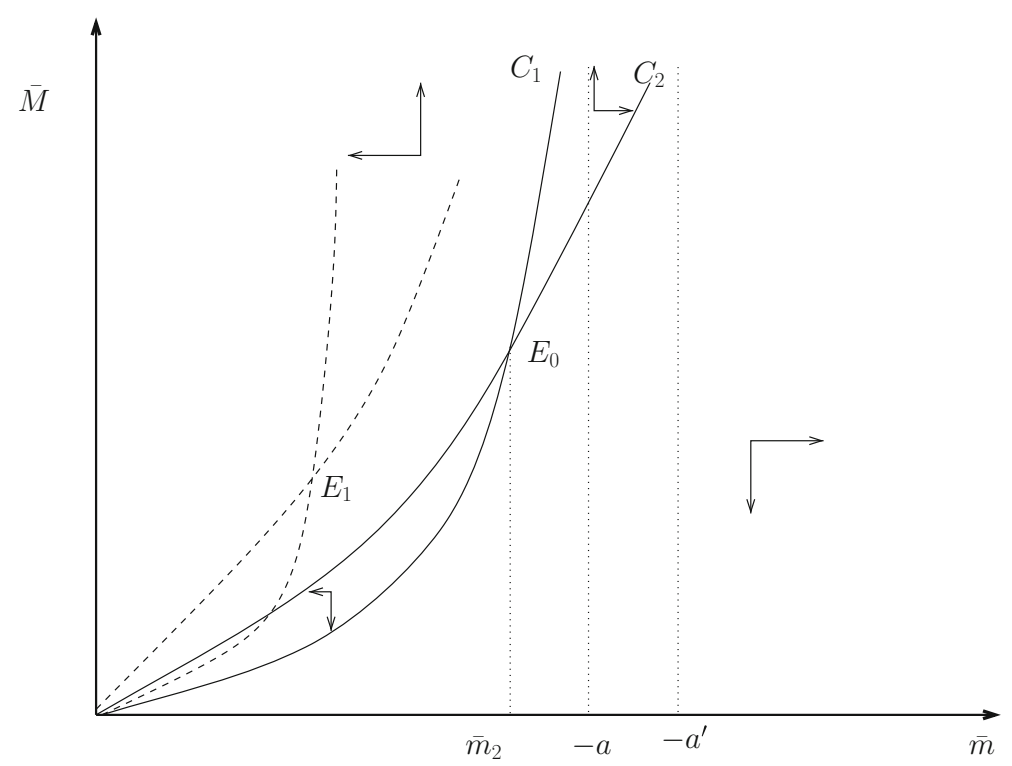

Fig. 6 Phase diagram for multiplicative non-separable preferences, plane $(\bar{M}, \bar{m})$

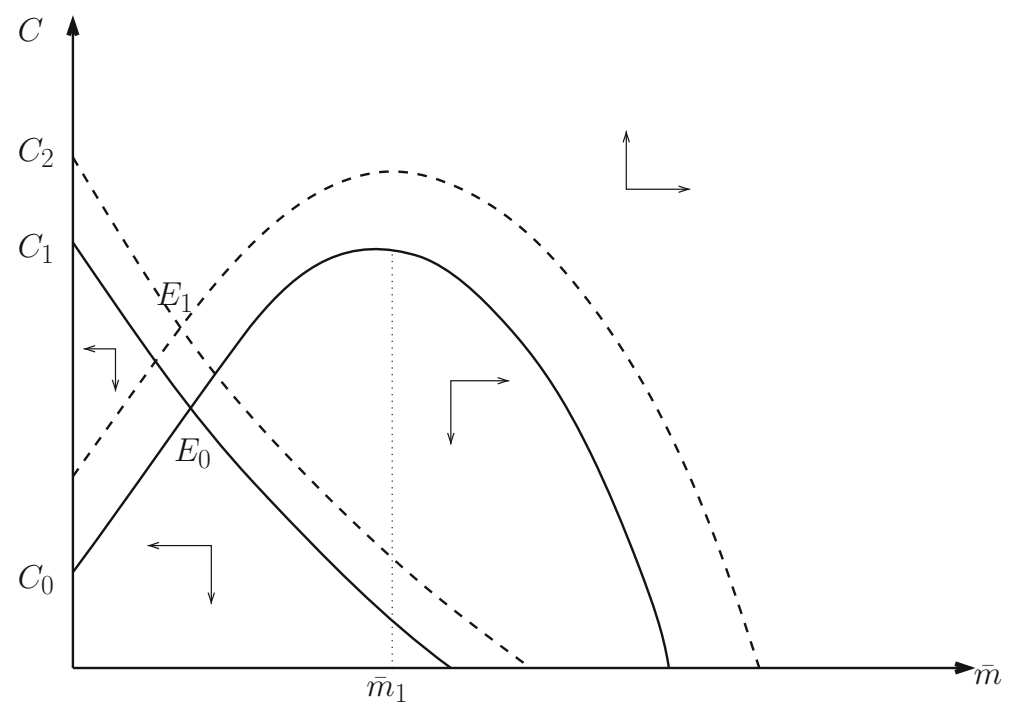

Fig. 7 Phase diagram for multiplicative non-separable preferences, plane $(C, \bar{m})$

but this reduction is mote than offset by the shift of consumption. The result is a net increase in health.

The second phase diagram (Fig. 6) is plotted onto the plane $(\bar{m}, \bar{M})$ by fixing $c$. The two curves are increasing with the variables $\bar{m}$ and $\bar{M}$, but the stock of health grows faster than the flow of investment. An increasing rate of health depreciation 
leads to a high reduction in the stock of health which is not fully compensated for by the investment. So the two curves shift to the left to yield the steady state $E_{1}$.

The third diagram (Fig. 7 ) is obtained onto the $(c, \bar{m})$ plane by fixing $\bar{M}$. There is a linear relation between $c$ and $\bar{m}$ that gives the monotonically decreasing curve. An increase in the depreciation rate shifts upward the consumption and the flow of investment in health on the curve $\dot{c}=0$. We have the same upward shift in the curve $\dot{\bar{m}}=0$. The final steady state is reached at $E_{1}$ where both variables increase.

Let us discuss insights from the equilibrium dynamics considering the effects of parameters in the different models and taking into account the dynamics of transition. We assume that the economy is on balanced growth path when a parameter varies, and analyze the adjustments to the new equilibrium path. Let us take the example of the phase diagram where we consider a variation in the rate of depreciation of health $\delta_{M}$. Fixing $\bar{m}$, the phase diagrams are obtained on the plane $(\dot{c}, \dot{M})$ of Figs. 2 and 5 wherein there are two curves for both the additive and multiplicative cases. In both cases, the $\dot{c}=0$ curves are independent of the stock of health. The curves $M=0$ are concave and grow with both variables. In the case of the separable utility, an increase in the rate of impairment of health implies a movement of the second curve $(\dot{M}=0)$ to the left. This induces a reduction in the stock of health by the move from the stable equilibrium $E_{0}$ to a new equilibrium $E_{1}$ where consumption remains constant. Conversely, in the multiplicative case, starting from the steady state $E_{0}$, an increase in $\delta_{M}$ shifts up the level of consumption, leading to the end point of equilibrium $E_{1}$. Impairment of health reduces the stock of health, but this decrease is more than offset by the increase in consumption. The result is a net increase in the stock of health.

Fixing $\bar{c}$ gives Figs. 3 and 6 on the $(\dot{m}, \dot{M})$ plane that relate the stock of health to health investment. For the additive as well as for the multiplicative case, the curve $(\dot{M}=0)$ increases, but the health stock increases faster than investment flows. When impairment of health increases, this implies a lower level of health stock. The curve $\dot{m}=0$ moves to the left. But the flow of investment increases, which means that the final steady state is reached when the curve $\dot{M}=0$ shifts to the right. The final state of equilibrium point is $E_{1}$ where the stock of health is lower than the first equilibrium point $E_{0}$.

Fixing $\bar{M}$ produces Figs. 4 and 7 in the $(\dot{c}, \dot{m})$ plane. For the additive case, starting from the equation $(\dot{c}=0)$, we see that there always exists a linear relationship between the flow of health investments and consumption. The trajectories converge to equilibrium from the areas of stability for values on the left of $E_{0}$. An increase in $\delta_{M}$ generates a high level of investment flows. In addition, a crowding out effect appears and consumption declines from $E_{0}$ to $E_{1}$. For the multiplicative case, the equilibrium is reached at point $E_{1}$ where both variables increase simultaneously.

\section{Convex combination of preferences}

In this section, we combine both cases in a general framework. Indeed, in between purely additive and multiplicative preferences, there might be a range of choices depending on parameter link that may drive agents' behavior. The welfare function $\mathscr{V}$ now takes the form: 


$$
\mathscr{V}(\cdot)=s U(C(z)) \varphi(M(z))+(1-s)[U(C(z))+\varphi(M(z))]
$$

and $s$ is a parameter link such that $0 \leq s \leq 1$. If $s=1$, then the individual preference becomes additive and, for $s=0$, it is multiplicative.

The dynamic system is then obtained by solving Eqs. (7a)-(7d). The optimization Eqs. (5a)-(5d) provide sufficient conditions for maximizing welfare because of the concavity of the utility function, the production of health and health investment. The Hamiltonian is a concave function of the state variables and control variables. The following result holds:

Proposition 4 The equilibrium values of health stock are located on a trajectory which is time dependent and given by the relation:

$$
\widehat{M}(z, s)=\left[\theta_{0}(s)+\theta_{1} e^{-z(r-\rho)}\right]^{\frac{1}{1-\sigma_{2}}}
$$

with $\theta_{0}(s)=-s \frac{1-\sigma_{2}}{1-s}$ and $\theta_{1}=\bar{\lambda}\left(1-\sigma_{2}\right)$ where $\bar{\lambda}$ is an integration constant.

Proof See "Appendix 1."

Proposition 4 is interesting as it shows that the balance of health variables $\widehat{M}$ and $\hat{m}$ can be expressed as a function of time, regardless of other real variables such as consumption and capital per capita. However, as Eqs. (7a)-(7d) of the general system establish the links between the variables in the model, the equilibrium expression of these variables can be recovered. But this approach is analytically complicated, if not impossible. Moreover, as we have documented in "Appendix 2," studying analytically the equilibrium dynamics of the model in the case of convex combination of preferences is unbearable.

The expression of health investment at equilibrium, $\widehat{m}(z)$, is then obtained:

$$
\widehat{m}(z)=\left(\frac{\delta_{M}}{b}\right)^{\frac{1}{\beta}}\left[\theta_{0}(s)+\theta_{1} \mathrm{e}^{-z(r-\rho)}\right]^{\frac{1}{\beta\left(1-\sigma_{2}\right)}}
$$

Relying on Eq. (7b) and using $S_{\varphi}\left[M^{*}(z)\right]=(1-s) \varphi_{M}\left[M^{*}(z)\right]$ and $S_{U}\left[C^{*}(z)\right]=$ $(1-s) U_{C}\left[C^{*}(z)\right]$, the expression of wage rate is obtained:

$$
w\left(M^{*}(z)\right)=\frac{\left(\delta_{M}+r\right) h_{m}\left[m^{*}(z)\right]}{\varphi_{M}\left[M^{*}(z)\right] \psi_{m}\left[m^{*}(z)\right]}-\frac{\varphi_{M}\left[M^{*}(z)\right]}{U_{C}^{2}\left[C^{*}(z)\right]}
$$

where $M^{*}(z)$ and $m^{*}(z)$ are replaced by their expressions.

Figure 8 shows the evolution of the stock of health and health investment. The curves depart from an initial value at time $z=0$ given by the expressions $\widehat{M}(0, s)$ and $\widehat{m}(0, s)$ as described below. As in the model of Ehrlich and Chuma (1990), $\widehat{M}$ and $\widehat{m}$ are decreasing and each tends to its minimum.

Furthermore, Proposition 4 allows us to study the limit behavior of stock and investment in health. We can distinguish two cases: (i) infinite horizon $(z \rightarrow \infty)$ 


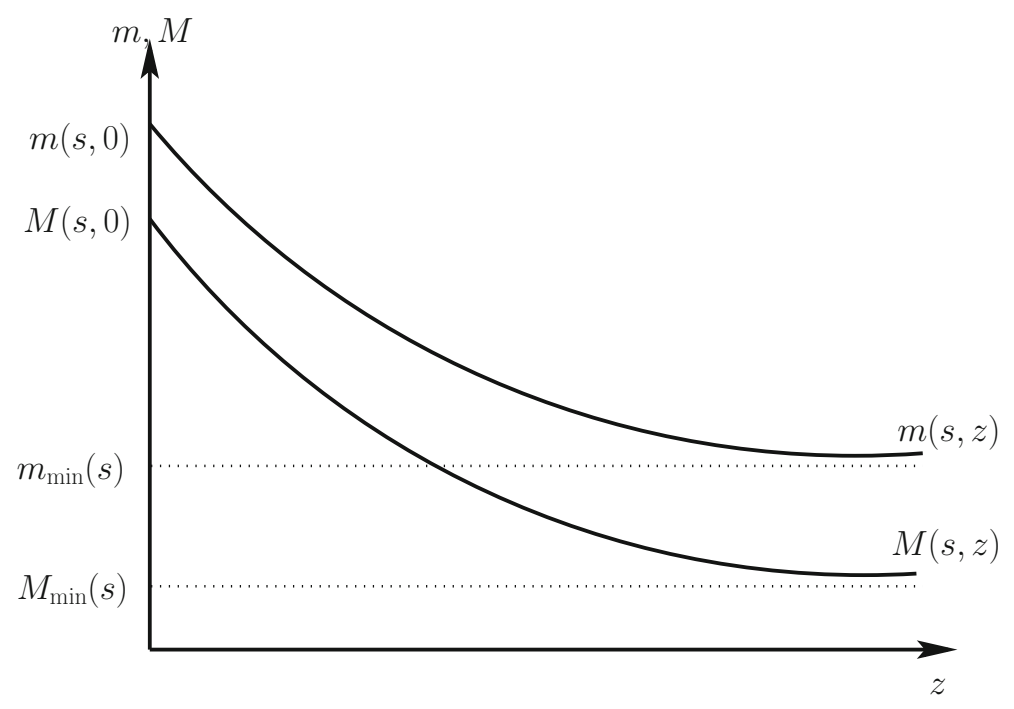

Fig. 8 Evolution of stock of health and health investment

and (ii) finite horizon $(z \rightarrow T)$. In the first case, taking the limit of Eqs. (33) and (34), we obtain, respectively: $\lim _{z \rightarrow \infty} \widehat{M}(z, s)=\widehat{M}(\infty, s)=\left[\theta_{0}(s)\right]^{\frac{1}{1-\sigma_{2}}}$ and $\lim _{z \rightarrow \infty} \widehat{m}(z, s)=\widehat{m}(\infty, s)=\left(\frac{\delta_{M}}{b}\right)^{\frac{1}{\beta}}\left[\theta_{0}(s)\right]^{\frac{1}{\beta\left(1-\sigma_{2}\right)}}$. For $z=0$, we have $\widehat{M}(0, s)=$ $\left[\theta_{0}(s)+\bar{\lambda}\left(1-\sigma_{2}\right)\right]^{\frac{1}{1-\sigma_{2}}}$ and $\widehat{m}(0, s)=\left(\frac{\delta_{M}}{b}\right)^{\frac{1}{\beta}}\left[\theta_{0}(s)+\bar{\lambda}\left(1-\sigma_{2}\right)\right]^{\frac{1}{\beta\left(1-\sigma_{2}\right)}}$. Infinite horizon also implies high health deterioration meaning that health will tend to its minimum. It follows that $\widehat{M}_{\min }(\infty, s)=\left[\theta_{0}(s)\right]^{\frac{1}{1-\sigma_{2}}}$ and $\widehat{m}_{\min }(\infty, s)=$ $\left(\frac{\delta_{M}}{b}\right)^{\frac{1}{\beta}}\left[\theta_{0}(s)\right]^{\frac{1}{\beta\left(1-\sigma_{2}\right)}}$.

The case of finite horizon is of particular interest because it provides the expression of the time limit for the stock of health to be low, and that life ends at some $T(\cdot, s)$. We have:

$$
\lim _{z \rightarrow T} \widehat{M}(z, s)=\widehat{M}(T, s)=\left[\theta_{0}(s)+\bar{\lambda}\left(1-\sigma_{2}\right) \mathrm{e}^{-T(r-\rho)}\right]^{\frac{1}{1-\sigma_{2}}}
$$

When $T$ is reached, health reaches its minimum given by Eq. (36). Figure 9 displays the graph for that case. $\widehat{M}(T, s)$ decreases and reaches its minimum at horizon $T(s)$.

An interesting theoretical issue is what would be the value of time horizon $T$, if the minimum level of health is known. Let us recall our approach to better understand the theoretical importance of the finite time horizon. In the model, we looked for the optimal paths of consumption, capital and health variables for infinite horizon. The framework of convex combination of preferences leads to purely temporal expression of health stock and health investment. These variables depend on what we call the structure parameter or convexity of the model, $s$. The latter allows us to balance the 


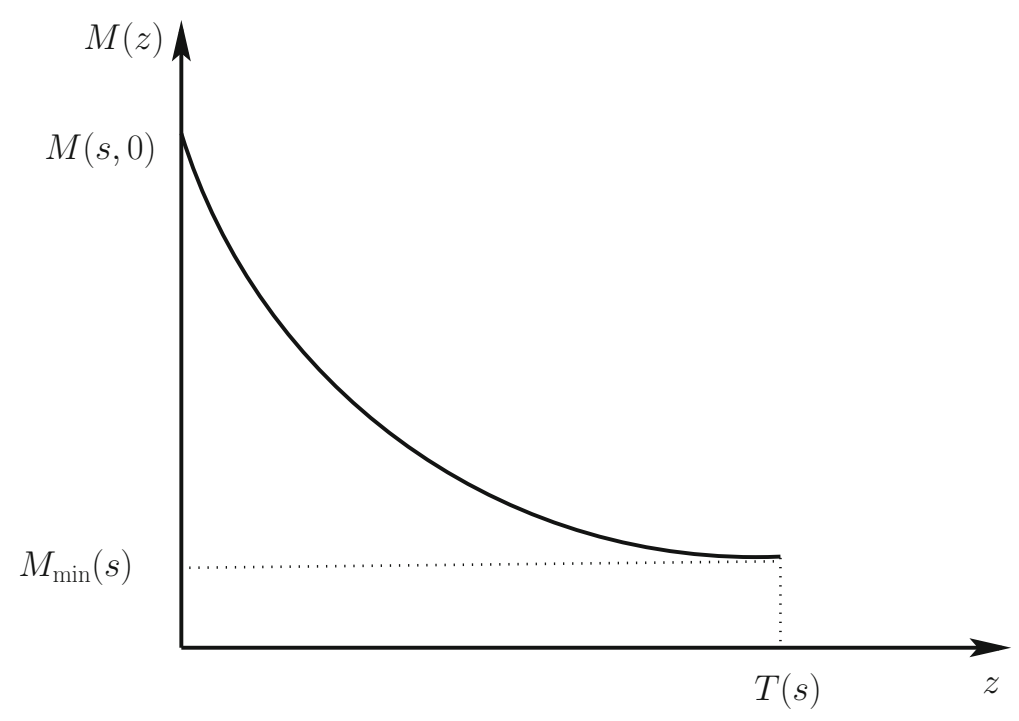

Fig. 9 Evolution of stock of health in finite horizon

model between the two polar cases: additive and multiplicative welfare functions. The benefit of having optimal variables that are expressed in terms of time is that one can identify the limits which in turn depend on the model parameters. Therefore, setting $s$, and making assumptions about the minimum value of the stock of health, we can infer a temporal horizon that represents longevity, i.e., life duration over which health keeps economic activities of work, consumption and investment. Suppose the minimum stock is not zero. Then, $T$ can be obtained from Eq. (36) for $\widehat{M}(T, s)=\widehat{M}_{\min }(s)$ and be expressed in terms of other model parameters as:

$$
T=\frac{1}{r-\rho} \ln \left(\frac{\bar{\lambda}\left(1-\sigma_{2}\right)}{\widehat{M}_{\min }(s)-\theta_{0}(s)}\right)=T_{0}+\frac{1}{r-\rho} \ln \left(\frac{1-\sigma_{2}}{\widehat{M}_{\min }(s)+s \frac{1-\sigma_{2}}{1-s}}\right)
$$

with $T_{0}=\frac{1}{r-\rho} \ln \bar{\lambda}$. The study of function $T$ with respect to parameters is interesting from two points of view. First, this horizon should ideally be the farthest as possible from zero. Therefore, it is crucial to understand the role of each parameter to achieve this goal. Secondly, we have not explicitly sought optimal longevity as in Ehrlich and Chuma (1990). However, our approach leads to a model of optimal lifetime which generalizes (Ehrlich and Chuma 1990). Indeed, as the authors, we find the same parameters that determine life span. In addition, here, life span also depends on the way welfare is chosen, i.e., parameter $s$. If we elaborate only on the effect of the parameter $\sigma_{2}$, we have the plot of $T$ in Fig. 10.

Here, $\bar{\sigma}_{2}=1-\frac{(1-s)}{2 s} \widehat{M}_{\min }(s), \overline{\bar{\sigma}}_{2}=1-\frac{(1-s)}{\bar{\lambda}(1-s)-s} \widehat{M}_{\min }(s)$ and $T\left(\bar{\sigma}_{2}, s\right)$ is given by Eq. (37) evaluated at $\bar{\sigma}_{2}$. Figure 10 also shows interesting aspects of the modeling. First of all, observe the vertical dotted line that stresses the constraint $\sigma_{2}<1$. The longevity $T$ curve shows a convex-concave shape. Indeed, the first portion of the curve which departs from $T(s, 0)$ decreases convexly to reach the inflection point $\left(T\left(\bar{\sigma}_{2}, s\right), \bar{\sigma}_{2}\right)$ 


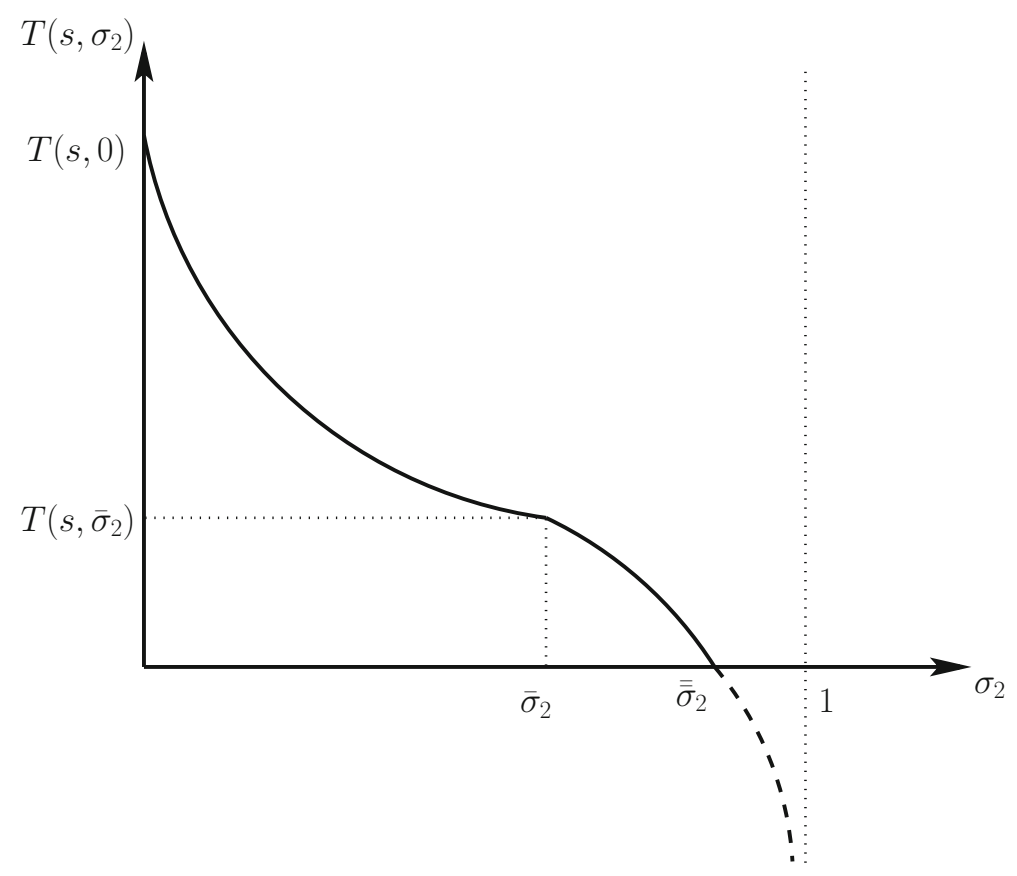

Fig. 10 Evolution of longevity

where the curve becomes concave till the point $\overline{\bar{\sigma}}_{2}$. Therefore, longevity is a decreasing function of the elasticity of inter-temporal substitution of health $\sigma_{2}$. We also see that as long as $\sigma_{2}<\overline{\bar{\sigma}}_{2}$, life span is strictly positive. This indicates that there is a maximal bound for $\sigma_{2}$ beyond which the stock of health is minimal. Thus, the choice of $\sigma_{2}$ will impact the longevity modeling. Indeed, if $\left.\sigma_{2} \in\right] \overline{\bar{\sigma}}_{2}, 1[$, longevity is zero. This means that the stock of health has no effect on real variables. As a result, there is no longer life: $\widehat{M}(z, s)=\widehat{M}_{\min }(s)=0$ for all $z$. However, if one chooses $\left.\sigma_{2} \in\right] \overline{\bar{\sigma}}_{2}, 1[$, it is still possible to give a positive value to $T$ provided that it is possible to identify the structural parameter $s$ which gives more weight to either the additive preference $(s \rightarrow 0)$ or the multiplicative one $(s \rightarrow 1)$. For $s=1$ (multiplicative), we have $\overline{\sigma_{2}}=\overline{\sigma_{2}}=1$. So one can choose $\sigma_{2}$ in the range [0,1[ and therefore $T>0$. In other words, opting for multiplicative preferences rules out the problem of existence of $T$. However, if an additive preference ( $s=0$ ) is chosen, one should care about the issue of existence of $T$.

Some comments related to Propositions 2-4 are in order. In economic theory, the effect of health care is often approached indirectly. Indeed, it is straightforward to measure health inputs and the indirect effects of health on the economy (through indicators that are positively correlated with good health conditions, such as increased life expectancy and low morbidity rates). Although the quality of health is not directly measurable, our model allows us to specify conditions for good health, by connecting three quantitative factors, namely health production, health investment and health costs. We have shown that the elasticities $\left(\alpha, \beta\right.$ and $\left.\sigma_{2}\right)$ of these three variables work together to define how health status influences labor. The model enables us to link the evolution of wage rates to health. The transmission belt between the wage rate and 


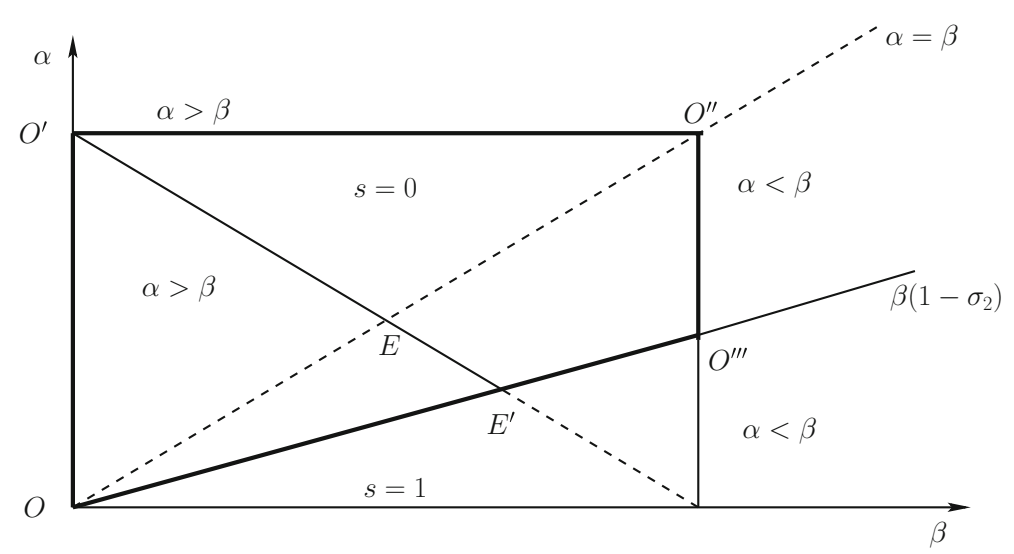

Fig. 11 Combination of elasticities

health is labor productivity. As better health conditions enhance human capacities, the values created in the production process are improved in turn.

Two problems arise: those of causality and indeterminacy. There is a causality problem because when wage rates become too high, the most productive agents, that is to say, those with highest wages, consume more and more goods that improve health. Thus, causality can pass from greater labor productivity to better health, not from health to productivity. This is typically a reverse causality issue. The indeterminacy arises with the model specification. Indeed, there are values of the elasticities $(\alpha=\beta)$ for which the model may not have equilibrium and the influence of health on the wage rate becomes indeterminate. This also coincides with the fact that the opportunity cost of health stock becomes time independent. Figure 11 shows the combination of parameters $\alpha$ and $\beta$ that allow our models to have solutions.

The axis $O O^{\prime \prime}$ makes an angle of $45^{\circ}$ with the axis $O \beta$. And the combination of solutions which have the properties of additive and multiplicative cases is delimited by the bold lines of the trapezium $\left(O O^{\prime} O^{\prime \prime} O^{\prime \prime \prime}\right)$, once the parameters $\alpha$ and $\beta$ are set. This is actually a combination of points belonging to the surfaces of triangles $\left(O O^{\prime} O^{\prime \prime}\right.$ (additive preferences) on the one hand and $O O^{\prime \prime} O^{\prime \prime \prime}$ (multiplicative preferences) on the other. These points are on the segment $O^{\prime} E^{\prime}$. However, the intersection point $E$ with the segment $O O^{\prime \prime}$ is excluded from the model because it fits the equality condition $\alpha=\beta$.

It is worth to notice a recurring problem in the field of health investment. Indeed, usually the aim is to look for the second best optimum in order to conciliate efficiency and social equity by proposing an optimal tax system and subsidies. Indeed, according to neoclassical theory, efficiency is reached when all agents behave competitively, and optimal allocations are then first best. Equity can be achieved through redistribution of income between healthy workers, workers whose health stock is low, and investors. This would have allowed us to disconnect the "final distribution of health stock" from that resulting from the ex-post prices and elasticities structure of production and investment. But at this stage of our model, the issue is not that of equity. Our intuition is that we could have come up with explanations for the difference in results between the additive and the multiplicative preferences. We leave this for future research. 


\section{Opportunity costs of health}

In this section, we compare the opportunity costs of health investment under the three alternative preferences. Such comparison is useful for policy analysis. Indeed, for a decision maker, it is interesting to know what investment alternative is the most effective in face of limited resources. Actually, for public policy reasons, the health sector is in competition with other economic sectors. As a result, the less costly alternative might be privileged. However, cost is only one input of the decision, as the latter should also consider the expected benefit from the investment in order to have a full picture of the decision options. This usually leads to an empirical cost-benefit analysis. Our objective here is to shed a theoretical light on the cost aspect of that mechanism.

In Sect. 2, we established in Eq. (9) the expression for the opportunity cost in a very general way. This provides the present value of the benefits of health stock available on the remaining life. We can derive the analogue of Eq. (9) for each type of preference. In the additive case, we have:

$$
g_{1}(M(z))=\int_{z}^{\infty}\left[\varphi_{M}(M(x))\left(w(M)+\frac{1}{\lambda_{A}(0)}\left(S_{\varphi} V_{S}\right) \mathrm{e}^{(\rho-r) x}\right)\right] \mathrm{e}^{-\left(\delta_{M}+r\right)(x-z)} \mathrm{d} x
$$

where the wage rate $w$ is given by Eq. (20c). For multiplicative preference, we have:

$$
g_{2}(M(z))=\int_{z}^{\infty}\left[\varphi_{M}(M(x))\left(w(M)+\frac{1}{\lambda_{A}(0)}\left(N_{\varphi} V_{N}\right) \mathrm{e}^{(\rho-r) x}\right)\right] \mathrm{e}^{-\left(\delta_{M}+r\right)(x-z)} \mathrm{d} x
$$

where the wage rate $w$ is given by Eq. (30). For the convex combination of preferences, we have:

$$
\begin{aligned}
& g_{3}(M(z)) \\
& \quad=\int_{z}^{\infty}\left[\varphi_{M}(M(x))\left(w(M)+\frac{1}{\lambda_{A}(0)}(s U+(1-s)) \mathrm{e}^{(\rho-r) x}\right)\right] \mathrm{e}^{-\left(\delta_{M}+r\right)(x-z)} \mathrm{d} x
\end{aligned}
$$

where the wage rate $w$ is given by Eq. (35). The functions or distributions $g_{1}(M(z)), g_{2}(M(z))$ and $g_{3}(M(z))$ for $0 \leq z \leq \infty$ can be compared using the notion of stochastic dominance. Define the distributions $g_{i}(M(x))$ for preference structure $i=1,2,3$ (additive, multiplicative and convex combination, respectively) as

$$
g_{i}(M(x))=\varphi_{M}(M(x))\left(w_{i}(M(x))+\frac{1}{\lambda_{A}(0)} \Phi_{i} \mathrm{e}^{(\rho-r) x}\right) \mathrm{e}^{-\left(\delta_{M}+r\right)(x-z)}
$$

where $\Phi_{1}=S_{\varphi} V_{S}, \Phi_{2}=N_{\varphi} V_{N}$ and $\Phi_{3}=s N_{\varphi} V_{N}+(1-s) S_{\varphi} V_{S}$. We can then write

$$
g_{i}\left(M((z))=\int_{z}^{\infty} g_{i}(M(x)) \mathrm{d} x\right.
$$


implying that $g_{i}(M(z))$ is the complementary cumulative distribution of $M(x)$. That is $g_{i}(M(z))=1-\mathscr{F}(M(z))$ where $\mathscr{F}(M(z))=\int_{-\infty}^{z} g_{i}(M(x)) d x$ is the cumulative distribution of $M(x)$.

Definition 1 A complementary cumulative distribution (CCD) $\mathscr{F}$ is said to first-order stochastically dominate (FOSD) another distribution $\mathscr{G}$ if and only if $\mathscr{F}(x) \geq \mathscr{G}(x)$ for all values of $x$. A CCD $\mathscr{F}$ is said to second-order stochastically dominate (SOSD) another distribution $\mathscr{G}$ if and only if $\int_{z}^{\infty} \mathscr{F}(x) \mathrm{d} x \geq \int_{z}^{\infty} \mathscr{G}(x) \mathrm{d} x$ for all $z$, with a strict inequality for at least some values of $z$.

Note that if $\mathscr{F}$ and $\mathscr{G}$ in Definition 1 were cumulative distributions rather than CCDs, then the inequalities would reverse. In relation to the expressions of opportunity costs, $g_{i}(M(z))$ FOSD $g_{j}(M(z))$ for $i \neq j$ implies that the opportunity cost under preference structure $i$ is greater than the opportunity cost under preference structure $j$ for all values of $M(z)$. The following result holds:

Proposition 5 Let the structure of opportunity costs be as in Eqs. (38-40) and let $\langle M(x)\rangle_{w_{i}}$ denote the expected value of $M(x)$ under wage rate distribution $w_{i}(M(x))$. If $C(x) \geq\left(1-\sigma_{1}\right)^{\frac{1}{1-\sigma_{1}}}$ for all $x$, then:

(i) $g_{2}(M(z))$ FOSD $g_{1}(M(z))$ whenever $w_{1}(M(x))=w_{2}(M(x))$ for all $M(x)$ or $\langle M(x)\rangle_{w_{2}} \geq\langle M(x)\rangle_{w_{1}}$

(ii) $g_{2}(M(z))$ FOSD $g_{3}(M(z))$ whenever $w_{2}(M(x))=w_{3}(M(x))$ for all $M(x)$ or $\langle M(x)\rangle_{w_{2}} \geq\langle M(x)\rangle_{w_{3}}$

(iii) $g_{3}(M(z))$ FOSD $g_{1}(M(z))$ whenever $w_{1}(M(x))=w_{3}(M(x))$ for all $M(x)$ or $\langle M(x)\rangle_{w_{3}} \geq\langle M(x)\rangle_{w_{1}}$.

\section{Proof See "Appendix 1."}

Remark 1 There are two possible ways to determine whether $\langle M(x)\rangle_{w_{i}} \geq\langle M(x)\rangle_{w_{j}}$ or to establish conditions on the model parameters for the inequality to be true. The first is to directly solve for the average values $\langle M(x)\rangle_{w_{i}}$ for all $i=1,2,3$ and compare the resulting functions. That is, for each $i,\langle M(x)\rangle_{w_{i}}=\int_{-\infty}^{\infty} M(x) w_{i}(M(x)) \mathrm{d} x$. The difficultly with this approach is that computing the respective integrals is not necessarily feasible, specially when the relationship between $w_{i}(M(x))$ and $M(x)$ is implicit as it is the case for the additive preferences (see Eq. 20c). Moreover, even when the integration is feasible, the resulting expressions are too complex for direct comparison. The second technique would be to solve for the value of $M(x)$ (denoted by $\left.M^{*}(x)\right)$ for which $w_{i}(M(x))=w_{j}(M(x))$. For example, in the case of $w_{1}(M(x))$ and $w_{2}(M(x)), M^{*}(x)$ can be obtained by solving the steady-state simultaneous equations. Once $M^{*}(x)$ is obtained, one could then obtain the derivative (tangent) of $w_{i}(M(x))$ at $M^{*}(x)$ for each $i$. The idea would then be to compare the resulting values, such that if the derivative of $w_{i}(M(x))$ at $M^{*}(x)$ is greater than the derivative of $w_{j}(M(x))$ at $M^{*}(x)$ then $\langle M(x)\rangle_{w_{i}} \geq\langle M(x)\rangle_{w_{j}}$. This approach is equally infeasible due to complexity of each $w_{i}(M(x))$.

Proposition 5 allows us to study the relative behavior of the opportunity costs for different types of modeling. Are these costs minimized or exaggerated, if an additive, 
multiplicative or a convex combination of the two is assumed? To answer this question, we have made assumptions about the wage rates to simplify calculations. We find that (i) the opportunity cost is higher in the multiplicative model than in the additive model, (ii) it is higher in the multiplicative model than in the convex combination model, (iii) it is higher in the convex combination model than in the additive model. The relationships described above are also based on the condition that consumption has a lower limit set defined by the terms of stochastic dominance.

To understand the intuition behind these results, it is worth noticing that in this general equilibrium setting in which labor productivity depends on health, any increase in health investment has an opportunity cost, at least in terms of consumption. Presumably if the inter-temporal substitution of health becomes stronger, it would contradict the gradual depreciation of the stock of health over time. Thus, substitutability cannot grow indefinitely, unless it is accompanied by higher investment costs, which explains the upper limit imposed on the elasticity of inter-temporal substitution.

In terms of policy implication, two contrasting visions appear. First, it is plausible to assume that opportunity costs (in terms of consumption) of investments in health are greater in developing countries than in developed countries, although the constraints on the impact of health on the wage rate appear to be identical for both. The multiplicative preferences are therefore more appropriate for developing countries where the inertia in health behaviors is much higher. Similarly, additive preferences that consider consumption and health spending as separable in the welfare function seem more appropriate for developed countries. The dilemma is that in order for health to have a strong influence on the wage rate and therefore on wage income, it must bear higher and higher costs of health investment. This calls for a trade-off which is not always easy for developing countries. A solution for these countries would be first to implement public policies aimed at facilitating access to low-cost health care. This implies increased cooperation with developed countries which have advanced social and health protection systems, while developing social security schemes. Fight against epidemics is a good illustration.

\section{Conclusion}

In this study, we develop a general equilibrium framework to study the role of preference structure. We connect the stock of health consumption, capital, wage rate, interest rate and parameters such as time preference and elasticities of inter-temporal substitution. We have shown that there is a unique value of the stock of health, which ensures the equilibrium dynamic of the economy, regardless of the form of the welfare function. We find that there are three parameters that play a crucial role not only for the existence of the equilibrium values, but also for the effect of health on the wage rate: the elasticity of substitution of health between two periods, the elasticity of investment in health and that of the dual cost of this investment relative to investment flows itself.

The main question we addressed is how the stock of health affects the wage rate. The answer is not straightforward as it might look at first glance. Indeed, if the preferences are additive, the effect of the stock of health on the wage rate is positive only under some parameter constraints defined by the optimization problem. We find that there 
is a reservation wages mechanism under play. If the reservation wage is less than the market wage, the effect of health on wages is positive. If the reservation wage is higher than the market wage, the effect of health on wages becomes negative. When the preferences are multiplicative, the effect of health on wages is positive everywhere.

Several challenges remain to be addressed. Some of them include investigating: (i) the role for preventive health care along with having the consumer's wage and working time dependent on health, (ii) the heterogeneity and population uncertainty (e.g., young/old, insured/uninsured), (iii) infectious diseases and their modes of transmission in our dynamic setting to study as to how health deterioration may affect the existence of solutions to the optimization problem as well to assess the impact of health on variables such as capital accumulation, consumption, wage rates and productivity.

Acknowledgments This study has immensely benefited from the comments of two anonymous referees and the co-editor of the Journal, Tim Kehoe. We gratefully acknowledge useful comments and stimulating remarks from Raouf Boucekkine, Noel Bonneuil, Matteo Cervellati, Miguel Perez-Nievas, Hippolyte d'Albis, Frédéric Dufourt, Daniel Opolot, Eleni Yitbarek and Hassen Wako. This work was supported by the FERDI (Fondation pour les Etudes et Recherches sur le Développement International) and the Agence Nationale de la Recherche of the French government through the program "Investissements d'avenir ANR10-LABX-14-01." Théophile Azomahou thanks the support of UNU-MERIT. The usual disclaimer applies.

Open Access This article is distributed under the terms of the Creative Commons Attribution 4.0 International License (http://creativecommons.org/licenses/by/4.0/), which permits unrestricted use, distribution, and reproduction in any medium, provided you give appropriate credit to the original author(s) and the source, provide a link to the Creative Commons license, and indicate if changes were made.

\section{Appendix 1: Proofs}

\section{Proof of Proposition 1}

The proof is quite intuitive. Indeed, let $w^{*}$ denote the equilibrium wage rate. The lefthand side of Eq. (20c) is strictly increasing in $\widehat{M}$, while the right-hand side is strictly decreasing, and the latter is equal to zero when

$$
\widehat{M}=\frac{b}{\delta_{M}}\left[\frac{w^{*}}{1-\epsilon}-\left(-w^{*}\right)^{\frac{1}{\sigma_{1}}}-\frac{w^{*} \epsilon}{(r+\delta)(1-\epsilon)}\right]^{\beta}
$$

and equals to $\frac{b\left(\frac{b}{\delta_{M}}\right)^{-\sigma_{2}} \beta}{\pi\left(\delta_{M}+r\right) \alpha}\left[w^{*}+\left(\frac{w^{*}}{1-\epsilon}\left(1-\frac{\epsilon}{r+\delta}\right)\right)^{\sigma_{1}}\right]$ if $\widehat{M}=0$, which implies that there is a unique solution $\widehat{M}$ to Eq. (20c).

\section{Proof of Proposition 2}

The proof of the first inequality $\alpha \geq \beta\left(1-\sigma_{2}\right)$ results from studying the variation of $w$ with respect to health stock $M$. Equation (20c) can be rewritten in terms of output as: 
$f(M)=\widehat{M}^{\sigma_{2}-1+\frac{\alpha}{\beta}}-\frac{b\left(\frac{b}{\delta_{M}}\right)^{-\sigma_{2}} \beta}{\pi\left(\delta_{M}+r\right) \alpha}\left[w+\left(\frac{w}{1-\epsilon}\left(1-\frac{\epsilon}{r+\delta}\right)-\left(\frac{\delta_{M}}{b}\right)^{\frac{1}{\beta}} \widehat{M}^{\frac{1}{\beta}}\right)^{\sigma_{1}}\right]$

Relying on the implicit function theorem, after rearranging the terms of the derivative below, one gets:

$$
\frac{\partial w(M)}{\partial M}=-\frac{\partial f(M) / \partial M}{\partial f(M) / \partial w(M)}=\frac{\mathscr{W}_{\mathscr{N}}}{\mathscr{W}_{\mathscr{D}}}
$$

where

$$
\begin{aligned}
\mathscr{W}_{\mathscr{N}}= & \left(\frac{b}{\delta_{M}}\right)^{\sigma_{2}} \pi\left(\delta_{M}+r\right) \alpha \\
& {\left[\frac{b\left(\frac{b}{\delta_{M}}\right)^{-\sigma_{2}}\left(\frac{\delta_{M}}{b}\right)^{\frac{1}{\beta}} M^{-1+\frac{1}{\beta}}\left(-\left(\frac{\delta_{M}}{b}\right)^{\frac{1}{\beta}} M^{\frac{1}{\beta}}+\frac{w\left(1-\frac{\epsilon}{r+\delta}\right)}{1-\epsilon}\right)^{-1+\sigma_{1}} \sigma_{1}}{\pi\left(\delta_{M}+r\right) \alpha}\right.} \\
& \left.+M^{-2+\frac{\alpha}{\beta}+\sigma_{2}}\left(-1+\frac{\alpha}{\beta}+\sigma_{2}\right)\right]
\end{aligned}
$$

and

$$
\mathscr{W}_{\mathscr{D}}=b \beta\left[1+\frac{\left(1-\frac{\epsilon}{r+\delta}\right)\left(-\left(\frac{\delta_{M}}{b}\right)^{\frac{1}{\beta}} M^{\frac{1}{\beta}}+\frac{w\left(1-\frac{\epsilon}{r+\delta}\right)}{1-\epsilon}\right)^{-1+\sigma_{1}} \sigma_{1}}{1-\epsilon}\right]
$$

It follows from $\mathscr{W}_{\mathscr{N}}$ and $\mathscr{W}_{\mathscr{D}}$ that the effect of the health stock on the wage rate is positive if $\alpha \geq \beta\left(1-\sigma_{2}\right)$. The proof of the second inequality $\alpha>\beta$ follows from the stability of equilibria. We introduce this in the following lemma.

Lemma 3 The dynamic system is stable iff $\alpha>\beta$ and $\alpha \geq \beta\left(1-\sigma_{2}\right)$.

Proof The proof of this lemma requires studying the determinant of the Jacobian matrix of the system (24a)-(24c). After deriving and rearranging terms of calculations, the Jacobian is given by

$$
\mathscr{J}=\left(\begin{array}{lll}
\mathscr{J}_{11} & \mathscr{J}_{12} & \mathscr{J}_{13} \\
\mathscr{J}_{21} & \mathscr{J}_{22} & \mathscr{J}_{23} \\
\mathscr{J}_{31} & \mathscr{J}_{32} & \mathscr{J}_{33}
\end{array}\right)
$$


where $\mathscr{J}_{11}=\mathscr{J}_{12}=\mathscr{J}_{31}=1, \mathscr{J}_{13}=0$ and

$$
\begin{aligned}
& \mathscr{J}_{21}=1-\frac{b c^{-1+\sigma_{1}} k^{\sigma_{1}-\sigma_{2}-\alpha+\beta} \bar{m}^{-\alpha+\beta} \bar{M}^{-\sigma_{2}} \sigma_{1} \beta}{\pi \alpha(\alpha-\beta)} \\
& \mathscr{J}_{22}=1-\frac{b k^{-\sigma_{2}-\alpha+\beta} \bar{m}^{-1-\alpha+\beta} \bar{M}^{-\sigma_{2}}\left(c^{\sigma_{1}} k^{\sigma_{1}}+w\right) \beta(-\alpha+\beta)}{\pi \alpha(\alpha-\beta)} \\
& \mathscr{J}_{23}=1-\frac{b k^{-\sigma_{2}-\alpha+\beta} \bar{m}^{-\alpha+\beta} \bar{M}^{-1-\sigma_{2}} \sigma_{2}\left(c^{\sigma_{1}} k^{\sigma_{1}}+w\right) \beta}{\pi \alpha(\alpha-\beta)} \\
& \mathscr{J}_{32}=1+\frac{b k^{-1+\beta} \bar{m}^{-1+\beta} \beta}{\bar{M}} \\
& \mathscr{J}_{33}=-\frac{b k^{-1+\beta} \bar{m}^{\beta}}{\bar{M}^{2}}
\end{aligned}
$$

The determinant of $\mathscr{J}$ is computed as:

$$
\begin{aligned}
\operatorname{det}(\mathscr{J})= & -\frac{1}{c \pi \alpha(\alpha-\beta)}\left[b ^ { 2 } k ^ { - 1 - \sigma _ { 2 } - \alpha + 2 \beta } \overline { m } ^ { - 1 - \alpha + 2 \beta } \overline { M } ^ { - 2 - \sigma _ { 2 } } \beta \left(c^{\sigma_{1}} k^{\sigma_{1}} \bar{m} \sigma_{1}\right.\right. \\
& \left.\left.+c^{1+\sigma_{1}} k^{\sigma_{1}}\left(\alpha+\left(-1+\sigma_{2}\right) \beta\right)+c w\left(\alpha+\left(-1+\sigma_{2}\right) \beta\right)\right)\right]
\end{aligned}
$$

which is negative if $\alpha>\beta$ to ensure the positivity of the denominator. This also implies the existence of a saddle point. The stability also depends on the sign of the trace of the matrix. The trace is computed as

$$
\operatorname{Tr}(\mathscr{J})=2-\frac{b k^{-1+\beta} \bar{m}^{\beta}}{\bar{M}^{2}}+\frac{b k^{-\sigma_{2}-\alpha+\beta} \bar{m}^{-1-\alpha+\beta} \bar{M}^{-\sigma_{2}}\left(c^{\sigma_{1}} k^{\sigma_{1}}+w\right) \beta}{\pi \alpha}
$$

which is positive.

\section{Proof of Proposition 3}

To prove (i), it is straightforward to check that the left-hand side of Eq. (29c) is increasing, whereas the right-hand side is decreasing. The inequality in (ii) can be split into two parts: $\alpha \geq \beta\left(1-\sigma_{2}\right)$ and $\alpha<\beta$. The proof of the first part relies on the study of Eq. (29c). Solving this relation with respect to wage leads to:

$$
w(M)=\frac{\left(\frac{\delta_{M}}{b}\right)^{\frac{\alpha}{\beta}} M^{-1+\sigma_{2}} \pi\left(\delta_{M}+r\right) \alpha\left(M^{\frac{\alpha}{\beta}}+\frac{\delta_{M}\left(\frac{\delta_{M}}{b}\right)^{-\frac{\alpha}{\beta}}\left(\frac{\delta_{M} M}{b}\right)^{\frac{1}{\beta}} \beta\left(-1+\sigma_{2}\right)}{\pi\left(\delta_{M}+r\right) \alpha\left(-1+\sigma_{1}\right)}\right)}{\delta_{M} \beta\left(1+\frac{M^{-1+\sigma_{2}}(2 r+2 \delta-\delta \epsilon)\left(-1+\sigma_{2}\right)}{(r+\delta)(1-\epsilon)\left(-1+\sigma_{1}\right)}\right)}
$$


The derivative of (45) is given by

$$
\frac{\partial w(M)}{\partial M}=\frac{\mathscr{W}_{\mathscr{N}}}{\mathscr{W}_{\mathscr{D}}}
$$

where

$$
\begin{aligned}
\mathscr{W}_{\mathscr{N}}= & M^{1+\alpha} \alpha\left(1+\frac{M^{-1+\sigma_{2}}(2 r+2 \delta-\delta \epsilon)\left(-1+\sigma_{2}\right)}{(r+\delta)(1-\epsilon)\left(-1+\sigma_{1}\right)}\right) \\
& +\left(\frac{M^{\sigma_{2}}(2 r+2 \delta-\delta \epsilon)\left(M^{\alpha}+\frac{\delta_{M}\left(\frac{\delta_{M}}{b}\right)^{-\alpha} \beta\left(-1+\sigma_{2}\right)^{2}}{\pi\left(\delta_{M}+r\right) \alpha\left(-1+\sigma_{1}\right)}\right)}{(r+\delta)(1-\epsilon)\left(-1+\sigma_{1}\right)}\right) \\
& +M\left(M^{\alpha}+\frac{\delta_{M}\left(\frac{\delta_{M}}{b}\right)^{-\alpha} \beta\left(-1+\sigma_{2}\right)}{\pi\left(\delta_{M}+r\right) \alpha\left(-1+\sigma_{1}\right)}\right) \\
& \times\left(1+\frac{M^{-1+\sigma_{2}}(2 r+2 \delta-\delta \epsilon)\left(-1+\sigma_{2}\right)}{(r+\delta)(1-\epsilon)\left(-1+\sigma_{1}\right)}\right)\left(-1+\frac{1}{\beta}+\sigma_{2}\right)
\end{aligned}
$$

and

$$
\mathscr{W}_{\mathscr{D}}=\delta_{M} \beta\left(1+\frac{M^{-1+\sigma_{2}}(2 r+2 \delta-\delta \epsilon)\left(-1+\sigma_{2}\right)}{(r+\delta)(1-\epsilon)\left(-1+\sigma_{1}\right)}\right)^{2}
$$

which proves the first inequality in (ii). The proof of the second inequality $\alpha<\beta$ follows from the stability of equilibria. We introduce the following lemma.

Lemma 4 The dynamical system is stable iff $\alpha<\beta$ and $\alpha \geq \beta\left(1-\sigma_{2}\right)$.

Proof The proof of this lemma requires studying the determinant of the Jacobian matrix of the system (31). After deriving and rearranging terms of calculations, the Jacobian is given by

$$
\mathscr{J}=\left(\begin{array}{lll}
\mathscr{J}_{11} & \mathscr{J}_{12} & \mathscr{J}_{13} \\
\mathscr{J}_{21} & \mathscr{J}_{22} & \mathscr{J}_{23} \\
\mathscr{J}_{31} & \mathscr{J}_{32} & \mathscr{J}_{33}
\end{array}\right)
$$

where $\mathscr{J}_{11}=\mathscr{J}_{31}=1$ and

$$
\begin{aligned}
& \mathscr{J}_{12}=1-\frac{b k^{1-\beta} \bar{m}^{-1+\beta} \beta\left(-1+\sigma_{2}\right)}{\bar{M} \sigma_{1}} \\
& \mathscr{J}_{13}=-\frac{k^{-\beta}\left(\delta_{M} \bar{M} k^{\beta}-b k \bar{m}^{\beta}\right)\left(-1+\sigma_{2}\right)}{M^{2} \sigma_{1}} \\
& \mathscr{J}_{21}=-\frac{\bar{M} \pi \alpha^{2}-b k^{1+\alpha-\beta} \bar{m}^{-\alpha+\beta} \beta-\bar{M} \pi \alpha \beta+\bar{M} \pi \alpha(-\alpha+\beta) \sigma_{1}+b k^{1+\alpha-\beta} \bar{m}^{-\alpha+\beta} \beta \sigma_{2}}{\bar{M} \pi \alpha(\alpha-\beta)\left(-1+\sigma_{1}\right)}
\end{aligned}
$$




$$
\begin{aligned}
\mathscr{J}_{22}= & \frac{1}{\pi \alpha\left(-1+\sigma_{1}\right)} k^{-\beta} \bar{m}^{-1-\alpha} M^{-1-\sigma_{2}}\left(-k^{\beta} \bar{m}^{1+\alpha} M^{1+\sigma_{2}} \pi \alpha-b c k^{1+\alpha} \bar{m}^{\beta} M^{\sigma_{2}} \beta\right. \\
& \left.-b k^{\alpha+\sigma_{2}} \bar{m}^{\beta} M w \beta+\bar{M}\left(-k^{\beta} \bar{m}^{1+\alpha} M^{\sigma_{2}} \pi \alpha-b k^{\alpha+\sigma_{2}} \bar{m}^{\beta}\right) \sigma_{1}+b c k^{1+\alpha} \bar{m}^{\beta} M^{\sigma_{2}} \beta \sigma_{2}\right) \\
\mathscr{J}_{23}= & \frac{b k^{\alpha-\beta} \bar{m}^{-\alpha+\beta} \bar{M}^{-2-\sigma_{2}} \beta\left(-c k \bar{M}^{2}+\left(c k \bar{M}^{2}-k^{\sigma_{2}} M w+k^{\sigma_{2}} M w \sigma_{1}\right) \sigma_{2}\right)}{\pi \alpha(\alpha-\beta)\left(-1+\sigma_{1}\right)} \\
\mathscr{J}_{32}= & 1+\frac{b k^{1+\beta} \bar{m}^{-1+\beta} \beta}{\bar{M}} \\
\mathscr{J}_{33}= & -\frac{b k^{1+\beta} \bar{m}^{\beta}}{\bar{M}^{2}}
\end{aligned}
$$

The determinant of $\mathscr{J}$ is computed as:

$$
\begin{aligned}
\operatorname{det}(\mathscr{J})= & \frac{(\alpha-\beta)}{\pi \alpha\left(1-\sigma_{1}\right) \sigma_{1}} b k^{-2 \beta} \bar{m}^{-1-\alpha+\beta} \bar{M}^{-4-\sigma_{2}} \beta \\
& \left(b k^{1+\alpha+2 \beta+\sigma_{2}} \bar{m}^{\beta} \bar{M}^{2} w \sigma_{1}^{2}\left(\alpha-\beta+\beta \sigma_{2}\right)\right. \\
& {\left[( 1 - \sigma _ { 2 } ) \left(b k^{1+\alpha} \bar{m}^{\beta} \bar{M}\left(k \bar{M}^{\sigma_{2}}(\bar{m}+c \alpha)+k^{\sigma_{2}} \bar{M} w\right)\right.\right.} \\
& -\delta_{M} \bar{M} k^{\beta}\left(k^{\alpha+\sigma_{2}} \bar{M}^{2} w+b k^{2+\alpha+\beta} \bar{m}^{\beta} \bar{M}^{\sigma_{2}} \beta\right. \\
& \left.+k^{1+\alpha} \bar{M}^{1+\sigma_{2}}(\bar{m}+c)\right)+k^{1+\alpha}\left(\delta_{M} \bar{M} k^{\beta} \bar{M}^{\sigma_{2}}\left(\bar{m} \bar{M}+c \bar{M}+b k^{1+\beta} \bar{m}^{\beta} \beta\right)\right. \\
& \left.\left.+b \bar{m}^{\beta} \bar{M}\left(-k \bar{M}^{\sigma_{2}}(\bar{m}+c \alpha)+k^{\sigma_{2}} \bar{M} w \beta\right)\right) \sigma_{2}\right) \\
& +\bar{M} \sigma_{1}\left(\delta_{M} \bar{M} k^{\beta}\left(-k^{\alpha+\sigma_{2}} \bar{M} w+k^{1+2 \beta} \bar{m}^{\alpha} \bar{M}^{\sigma_{2}} \pi \alpha\right)\right. \\
& b k^{1+\beta} \bar{m}^{\beta}\left(k^{1+2 \beta} \bar{M}^{\sigma_{2}}(\bar{m}+c \alpha)+k^{\sigma_{2}} \bar{M}^{2 \beta} w k^{2 \beta+\sigma_{2}} \bar{M} w\right) \\
& +\left(\delta_{M} \bar{M} k^{\beta}\left(-k^{\alpha+\sigma_{2}} \bar{M} w+k^{1+2 \beta} \bar{m}^{\alpha} \bar{M}^{\sigma_{2}} \pi \alpha\right)\right. \\
& \left.+b k^{1+\beta} \bar{m}^{\beta}\left(k^{1+2 \beta} \bar{M}^{\sigma_{2}}(\bar{m}+c \alpha)+k^{\sigma_{2}} \bar{M} w(\alpha-2 \beta)-k^{2 \beta+\sigma_{2}} \bar{M} w\right)\right) \sigma_{2} \\
& \left.\left.\left.+b k^{1+\alpha+\sigma_{2}} \bar{m}^{\beta} \bar{M} w \beta \sigma_{2}^{2}\right)\right)\right]
\end{aligned}
$$

which is negative if $\alpha<\beta$ to guarantee the positivity of the denominator. This also implies the existence of a saddle point. The stability depends on the sign of the trace of the matrix. It is computed as:

$$
\begin{aligned}
\operatorname{Tr}(\mathscr{J})= & \frac{1}{\pi \alpha\left(1-\sigma_{1}\right)}\left(2 \pi \alpha-\frac{b k^{1+\beta} \bar{m}^{\beta} \pi \alpha}{\bar{M}^{2}}\right. \\
& +\frac{b c k^{1+\alpha-\beta} \bar{m}^{-1-\alpha+\beta} \beta}{M}+b k^{\alpha-\beta+\sigma_{2}} \bar{m}^{-1-\alpha+\beta} \bar{M}^{-\sigma_{2}} w \beta
\end{aligned}
$$




$$
\begin{aligned}
& +\left(\left(-2+\frac{b k^{1+\beta} \bar{m}^{\beta}}{\bar{M}^{2}}\right) \pi \alpha-b k^{\alpha-\beta+\sigma_{2}} \bar{m}^{-1-\alpha+\beta} \bar{M}^{-\sigma_{2}} w \beta\right) \sigma_{1} \\
& \left.-\frac{b c k^{1+\alpha-\beta} \bar{m}^{-1-\alpha+\beta} \beta \sigma_{2}}{\bar{M}}\right)
\end{aligned}
$$

which is positive.

\section{Proof of Proposition 4}

The proof follows from the general system. Indeed, Eq. (7a) in the system becomes a differential equation of the form:

$$
s(r-\rho)+\frac{(1-s)(r-\rho) M(z)^{1-\sigma_{2}}}{1-\sigma_{2}}+(1-s) M(z)^{-\sigma_{2}} \dot{M}(z)=0
$$

This differential equation is linear in $\dot{M}(z)$, and it is straightforward to check that the analytical solution is given by the expression of $\widehat{M}(z, s)$ (Eq. 33) in the proposition.

\section{Proof of Proposition 5}

The proof follows from pairwise comparison of $g_{i}(M(z))$ 's. Generally,

$$
\begin{aligned}
g_{i}(M(z))-g_{j}(M(z))= & \int_{z}^{\infty} \varphi_{M}(M(x))\left(w_{i}(M(x))-w_{j}(M(x))\right. \\
& \left.\quad+\frac{1}{\lambda_{A}(0)}\left(\Phi_{i}-\Phi_{j}\right) \mathrm{e}^{(\rho-r) x}\right) \mathrm{e}^{-\left(\delta_{M}+r\right)(x-z)} \mathrm{d} x
\end{aligned}
$$

implying that the crucial components when performing pairwise comparisons are $w_{i j}(M(x))=w_{i}(M(x))-w_{j}(M(x))$ and $\Phi_{i j}(z)=\Phi_{i}-\Phi_{j}$. Since $\varphi_{M}(M(x)) \geq 0$ for all $M(x)$, it follows that $g_{i}(M(z))-g_{j}(M(z)) \geq 0$ (that is $g_{i}(M(z))$ FOSD $g_{i}(M(z))$ if either; $(a) w_{i j}(M(x))>0$ and $\Phi_{i j}(x) \geq 0$ for all $x$ hence all $M(x)$, or $(b) w_{i j}(M(x))=0$ and $\Phi_{i j}(x) \geq 0$ for all $x$ hence all $M(x),(c)$ or $\Phi_{i j}(x) \geq 0$ for all $x$ and there exists an $x^{*}$, and hence $M\left(x^{*}\right)$, such that $w_{i j}(M(x))>0$ for all $M(x) \geq M\left(x^{*}\right)$.

If $\langle M(x)\rangle_{w_{i}}$ is the expected value of $M(x)$ under wage rate distribution $w_{i}(M(x))$, the conditions that $w_{i j}(M(x))>0$ for all $M(x)$ and that there exists an $M\left(x^{*}\right)$ such that $w_{i j}(M(x))>0$ for all $M(x) \geq M\left(x^{*}\right)$, are equivalent to saying that $\langle M(x)\rangle_{w_{i}} \geq$ $\langle M(x)\rangle_{w_{j}}$. The first condition for $g_{i}(M(z))$ to FOSD $g_{j}(M(z))$ is then that $\langle M(x)\rangle_{w_{i}} \geq$ $\langle M(x)\rangle_{w_{j}}$. What remains is to show the condition under which $\Phi_{i j}(x) \geq 0$ for each pair of $i$ and $j$.

(i) For the case of additively separable and multiplicative preferences, we have that 


$$
\Phi_{21}(x)=N_{\varphi} V_{N}-S_{\varphi} V_{S}
$$

From the expression of $V, N$ and $S$, we have that $V_{N}=V_{S}=S_{\varphi}=1$ and $N_{\varphi}=U(C(x))$, such that $N_{\varphi} V_{N}=U(C(x))$ and $S_{\varphi} V_{S}=1 . \Phi_{21}(x)$ then becomes

$$
\Phi_{21}(x)=U(C(x))-1=\frac{1}{1-\sigma_{2}} C(x)^{1-\sigma_{2}}-1,
$$

implying that $\Phi_{21}(x) \geq 0$ if $C(x) \geq\left(1-\sigma_{1}\right)^{\frac{1}{1-\sigma_{1}}}$ for all $x$.

(ii) Similarly, for the multiplicative and convex combination preferences,

$$
\begin{aligned}
\Phi_{23}(x) & =N_{\varphi} V_{N}-\left(s N_{\varphi} V_{N}+(1-s) S_{\varphi} V_{S}\right)=(1-s) U(C(x))-(1-s) \\
& =(1-s)(U(C(x))-1)
\end{aligned}
$$

Since $0<s<1$, it follows that $\Phi_{23}(x) \geq 0$ if $U(C(x))-1 \geq 0$ for all $x \Rightarrow C(x) \geq\left(1-\sigma_{1}\right)^{\frac{1}{1-\sigma_{1}}}$ for all $x$.

(iii) Finally, for the convex combination and additive preferences,

$$
\Phi_{31}(x)=s N_{\varphi} V_{N}+(1-s) S_{\varphi} V_{S}-S_{\varphi} V_{S}=s(U(C(x))-1)
$$

Hence, $\Phi_{31}(x) \geq 0$ if $C(x) \geq\left(1-\sigma_{1}\right)^{\frac{1}{1-\sigma_{1}}}$ for all $x$.

\section{Appendix 2: Note on the convex combination of preferences}

For the convex combination of preferences, the steady state of the model is governed by the system:

$$
\begin{aligned}
\frac{\dot{\hat{C}}(z)}{\hat{C}(z)}= & \frac{(-1+s)(r-\rho) M(z)+s(r-\rho) M(z)^{\sigma_{2}}\left(-1+\sigma_{2}\right)-(-1+s)\left(-1+\sigma_{2}\right)\left(-\delta_{M} M(z)+b m(z)^{\beta}\right)}{\sigma_{1}\left((-1+s) M(z)+s M(z)^{\sigma_{2}}\left(-1+\sigma_{2}\right)\right)} \\
\frac{\dot{\hat{m}}(z)}{\hat{m}(z)}= & C(z)^{\sigma_{1}} m(z)^{1-\alpha} M(z)^{\sigma_{2}}\left(b \beta m ( z ) ^ { - 1 + \beta } M ( z ) ^ { - \sigma _ { 2 } } \left(-s-\frac{(-1+s) C(z)^{1-\sigma_{1}}}{-1+\sigma_{1}}\right.\right. \\
& \left.+w C(z)^{-\sigma_{1}}\left(-s-\frac{(-1+s) M(z)^{1-\sigma_{2}}}{-1+\sigma_{2}}\right)\right)+\pi\left(-\delta_{M}-r\right) \alpha C(z)^{-\sigma_{1}} m(z)^{-1+\alpha} \\
& \left.\left.\times\left(-s-\frac{(-1+s) M(z)^{1-\sigma_{2}}}{-1+\sigma_{2}}\right)\right)\left(-1+\sigma_{2}\right)\right) /\left(\pi \alpha(\alpha-\beta)\left((-1+s) M(z)+s M(z)^{\sigma_{2}}\left(-1+\sigma_{2}\right)\right)\right) \\
\dot{\hat{M}}(z)= & -\delta_{M}+\frac{b m(z)^{\beta}}{m(z)} \\
\dot{\hat{k}}_{z}= & B(M(z)) \hat{k}(z)^{\epsilon}-\hat{C}(z)-\hat{m}(z)-\delta \hat{k}(z)
\end{aligned}
$$


with $\hat{k}(0)$ and $\hat{M}(0)$ given, plus the transversality conditions. The steady-state values $\hat{C}, \hat{m}, \hat{M}$ and $\hat{k}$ can be obtained by equalizing $\dot{\hat{C}}, \dot{\hat{m}}, \dot{\hat{M}}, \dot{\hat{k}}$ to zero. The dynamics of the economy is driven at the equilibrium by the following system:

$$
\begin{aligned}
\frac{\dot{c}}{c}= & \frac{(-1+s)(r-\rho) \bar{M} k^{-1}+s(r-\rho) \bar{M}^{\sigma_{2}} k^{-\sigma_{2}}\left(-1+\sigma_{2}\right)-(-1+s)\left(-1+\sigma_{2}\right)\left(-\delta_{M} \bar{M} k^{-1}+b \bar{m}^{\beta} k^{-\beta}\right)}{\sigma_{1}\left((-1+s) \bar{M} k^{-1}+s \bar{M}^{\sigma_{2}} k^{-\sigma_{2}}\left(-1+\sigma_{2}\right)\right)} \\
& -\frac{r+\delta(1-\epsilon)}{\epsilon}+c+\bar{m} \\
\frac{\dot{m}}{m}= & C^{\sigma_{1}} \bar{m}^{1-\alpha} \bar{M}^{\sigma_{2}} k^{-\sigma_{2}+\alpha-1}\left(\left(b \beta \overline { m } ^ { - 1 + \beta } \overline { M } ^ { - \sigma _ { 2 } } k ^ { \sigma _ { 2 } - \beta + 1 } \left(-s-\frac{(-1+s) C^{1-\sigma_{1}}}{-1+\sigma_{1}}\right.\right.\right. \\
& \left.+w C^{-\sigma_{1}}\left(-s-\frac{(-1+s) \bar{M}^{1-\sigma_{2}} k^{\sigma_{2}-1}}{-1+\sigma_{2}}\right)\right)+\pi\left(-\delta_{M}-r\right) \alpha C^{-\sigma_{1} \bar{m}^{-1+\alpha} k^{1-\alpha}} \\
& \left.\left.\times\left(-s-\frac{(-1+s) \bar{M}^{1-\sigma_{2}} k^{\sigma_{2}-1}}{-1+\sigma_{2}}\right)\right)\left(-1+\sigma_{2}\right)\right) / \\
& \left(\pi \alpha(\alpha-\beta)\left((-1+s) \bar{M} k^{-1}+s M^{\sigma_{2}} k^{-\sigma_{2}}\left(-1+\sigma_{2}\right)\right)\right) \\
& -\frac{r+\delta(1-\epsilon)}{\epsilon}+c+\bar{m} \\
\frac{\dot{M}}{M}= & -\delta_{M}+\frac{b \bar{m}^{\beta} k^{\beta+1}}{\bar{M}}-\frac{r+\delta(1-\epsilon)}{\epsilon}+c+\bar{m}
\end{aligned}
$$

The Jacobian of this system is given by

$$
\mathscr{J}=\left(\begin{array}{lll}
\mathscr{J}_{11} & \mathscr{J}_{12} & \mathscr{J}_{13} \\
\mathscr{J}_{21} & \mathscr{J}_{22} & \mathscr{J}_{23} \\
\mathscr{J}_{31} & \mathscr{J}_{32} & \mathscr{J}_{33}
\end{array}\right)
$$

where $\mathscr{J}_{11}=\mathscr{J}_{13}=\mathscr{J}_{21}=\mathscr{J}_{31}=1$ and

$$
\begin{aligned}
\mathscr{J}_{12}= & 1-\frac{b k^{1-\beta} \bar{m}^{-1+\beta}(-1+s) \beta\left(-1+\sigma_{2}\right)}{\sigma_{1}\left(\frac{\bar{M}(-1+s)}{k}+k^{-\sigma_{2}} \bar{M}^{\sigma_{2}} s(-1+s)\right)} \\
\mathscr{J}_{13}= & -\frac{k^{-\beta}\left(\delta_{M} \bar{M} k^{\beta}-b k \bar{m}^{\beta}\right)\left(-1+\sigma_{2}\right)}{M^{2} \sigma_{1}} \\
\mathscr{J}_{22}= & \left(k ^ { - \beta } \overline { m } ^ { - 1 - \alpha } \overline { M } ^ { - \sigma _ { 2 } } \left(b k^{1+\alpha+\sigma_{2}} \bar{m}^{\beta} \bar{M}^{\sigma_{2}}(-1+s) \beta C\left(-1+\sigma_{2}\right)\right.\right. \\
& +b k^{1+\alpha+\sigma_{2}} \bar{m}^{\beta} \bar{M}^{\sigma_{2}} s \beta C^{\sigma_{1}}\left(-1+\sigma_{1}\right)\left(-1+\sigma_{2}\right)+\left(k^{\beta} \bar{m}^{1+\alpha} \bar{M}^{\sigma_{2}} \pi \alpha\right. \\
& \left.\left.\left.+b k^{\alpha+\sigma_{2}} \bar{m}^{\beta} w \beta\right)\left(-1+\sigma_{1}\right)\left(k^{\sigma_{2}} M(-1+s)-k \bar{M}^{\sigma_{2}} s+k \bar{M}^{\sigma_{2}} s \sigma_{2}\right)\right)\right) / \\
& \left(\pi \alpha\left(-1+\sigma_{1}\right)\left(k^{\sigma_{2}} \bar{M}(-1+s)-k M^{\sigma_{2}} s+k \bar{M}^{\sigma_{2}} s \sigma_{2}\right)\right) \\
\mathscr{J}_{23}= & \left(b k ^ { \alpha - \beta + \sigma _ { 2 } } \overline { m } ^ { - \alpha + \beta } \overline { M } ^ { - 1 - \sigma _ { 2 } } \beta \left(w\left(-1+\sigma_{1}\right) \sigma_{2}\left(k^{\sigma_{2}} \bar{M}(-1+s)-k \bar{M}^{\sigma_{2}} s+k \bar{M}^{\sigma_{2}} s \sigma_{2}\right)^{2}\right.\right. \\
& +k \bar{M}^{\sigma_{2}}(-1+s) C\left(-1+\sigma_{2}\right)\left(k^{\sigma_{2}} \bar{M}(-1+s)-k \bar{M}^{\sigma_{2}} s \sigma_{2}+k \bar{M}^{\sigma_{2}} s \sigma_{2}^{2}\right)
\end{aligned}
$$




$$
\begin{aligned}
& \left.\left.+k \bar{M}^{\sigma_{2}} s C^{\sigma_{1}}\left(-1+\sigma_{1}\right)\left(-1+\sigma_{2}\right)\left(k^{\sigma_{2}} M(-1+s)-k \bar{M}^{\sigma_{2}} s \sigma_{2}+k M^{\sigma_{2}} s \sigma_{2}^{2}\right)\right)\right) \\
& /\left(\pi \alpha(\alpha-\beta)\left(-1+\sigma_{1}\right)\left(k^{\sigma_{2}} \bar{M}(-1+s)-k \bar{M}^{\sigma_{2}} s+k \bar{M}^{\sigma_{2}} s \sigma_{2}\right)^{2}\right) \\
\mathscr{J}_{32}= & 1+\frac{b k^{1+\beta} \bar{m}^{-1+\beta} \beta}{\bar{M}} \\
\mathscr{J}_{33}= & -\frac{b k^{1+\beta} \bar{m}^{\beta}}{\bar{M}^{2}}
\end{aligned}
$$

The determinant of $\mathscr{J}$ is computed as:

$$
\begin{aligned}
& \operatorname{det}(\mathscr{J})=\frac{1}{M^{2}} k^{-2 \beta}\left(\left(k^{\sigma_{2}} m^{-1-\alpha}\left(\delta_{M} M k^{\beta}-b k m^{\beta}\right) M^{1-\sigma_{2}}(-1+s)\left(-1+\sigma_{2}\right)\right.\right. \\
& \left(k^{\sigma_{2}} M(-1+s)-k M^{\sigma_{2}} s \sigma_{2}+k M^{\sigma_{2}} s \sigma_{2}^{2}\right)\left(b k^{1+\alpha+\sigma_{2}} m^{\beta} M^{\sigma_{2}}(-1+s)\right. \\
& \beta C(z)\left(-1+\sigma_{2}\right)+b k^{1+\alpha+\sigma_{2}} m^{\beta} M^{\sigma_{2}} s \beta C(z)^{\sigma_{1}}\left(-1+\sigma_{1}\right)\left(-1+\sigma_{2}\right) \\
& +\left(k^{\beta} m^{1+\alpha} M^{\sigma_{2}} \pi \alpha+b k^{\alpha+\sigma_{2}} m^{\beta} w \beta\right)\left(-1+\sigma_{1}\right)\left(k^{\sigma_{2}} M(-1+s)-k M^{\sigma_{2}} s\right. \\
& \left.\left.\left.+k M^{\sigma_{2}} s \sigma_{2}\right)\right)\right) /\left(\pi \alpha\left(-1+\sigma_{1}\right) \sigma_{1}\left(k^{\sigma_{2}} M(-1+s)-k M^{\sigma_{2}} s+k M^{\sigma_{2}} s \sigma_{2}\right)^{3}\right) \\
& +\left(b k^{\alpha+\beta+\sigma_{2}} m^{-\alpha+\beta} M^{1-\sigma_{2}} \beta\left(1-\frac{b k^{-\beta} m^{-1+\beta}(-1+s) \beta\left(-1+\sigma_{2}\right)}{\sigma_{1}\left(\frac{M(-1+s)}{k}+k^{-\sigma_{2}} M^{\sigma_{2}} s\left(-1+\sigma_{2}\right)\right.}\right)\right. \\
& \left(w\left(-1+\sigma_{1}\right) \times \sigma_{2}\left(\left(k^{\sigma_{2}} M(-1+s)-k M^{\sigma_{2}} s+k M^{\sigma_{2}} s \sigma_{2}\right)^{2}\right.\right. \\
& +k M^{\sigma_{2}}(-1+s) C(z)\left(-1+\sigma_{2}\right)\left(k^{\sigma_{2}} M(-1+s)-k M^{\sigma_{2}} s \sigma_{2}+k M^{\sigma_{2}} s \sigma_{2}^{2}\right) \\
& \left.\left.+k M^{\sigma_{2}} s C(z)^{\sigma_{1}}\left(-1+\sigma_{1}\right)\left(-1+\sigma_{2}\right)\left(k^{\sigma_{2}} M(-1+s)-k M^{\sigma_{2}} s \sigma_{2}+k M^{\sigma_{2}} s \sigma_{2}^{2}\right)\right)\right) \\
& /\left(\pi \alpha(\alpha-\beta)\left(-1+\sigma_{1}\right)\left(k^{\sigma_{2}} M(-1+s)-k M^{\sigma_{2}} s+k M^{\sigma_{2}} s \sigma_{2}\right)^{2}\right)-b k^{1+3 \beta} m^{\beta} \\
& \times\left(1-\frac{b k^{-\beta} m^{-1+\beta}(-1+s) \beta\left(-1+\sigma_{2}\right)}{\sigma_{1}\left(\frac{M(-1+s)}{k}+k^{-\sigma_{2}} M^{\sigma_{2}} s\left(-1+\sigma_{2}\right)\right.}+\left(k^{-\beta} m^{-1-\alpha} M^{\sigma_{2}}\right.\right. \\
& \left(b k^{1+\alpha+\sigma_{2}} m^{\beta} M^{\sigma_{2}}(-1+s) \beta C(z) \times\left(-1+\sigma_{2}\right)+b k^{1+\alpha+\sigma_{2}} m^{\beta} M^{\sigma_{2}} s\right. \\
& \beta C(z)^{\sigma_{1}}\left(-1+\sigma_{1}\right)\left(-1+\sigma_{2}\right)+\left(k^{\beta} m^{1+\alpha} M^{\sigma_{2}} \pi \alpha+b k^{\alpha+\sigma_{2}} m^{\beta} w \beta\right) \\
& \left.\left.\times\left(-1+\sigma_{1}\right)\left(k^{\sigma_{2}} M(-1+s)-k M^{\sigma_{2}} s+k M^{\sigma_{2}} s \sigma_{2}\right)\right)\right) \\
& \left./\left(\pi \alpha\left(-1+\sigma_{1}\right)\left(k^{\sigma_{2}} M(-1+s)-k M^{\sigma_{2}} s+k M^{\sigma_{2}} s \sigma_{2}\right)\right)\right) \\
& -\left(k^{\beta+\sigma_{2}} M\left(1+\frac{\left.\left.b k^{1+\beta} m^{-1+\beta}\right) \beta\right)}{M}\right)\right. \\
& \left(\frac{\left(\delta_{M} M k^{\beta}-b k m^{\beta}\right)(-1+s)\left(-1+\sigma_{2}\right)\left(k^{\sigma_{2}} M(-1+s)-k M^{\sigma_{2}} s \sigma_{2}+k M^{\sigma_{2}} s \sigma_{2}^{2}\right)}{\sigma_{1}}\right. \\
& +1 /\left(\pi \alpha(\alpha-\beta)\left(-1+\sigma_{1}\right)\right) b k^{\alpha} m^{-\alpha+\beta} M^{-\sigma_{2}} \beta\left(w ( - 1 + \sigma _ { 1 } ) \sigma _ { 2 } \left(k^{\sigma_{2}} M(-1+s)\right.\right. \\
& \left.-k M^{\sigma_{2}} s+k M^{\sigma_{2}} s \sigma_{2}\right) 2+k M^{\sigma_{2}}(-1+s) C(z)\left(-1+\sigma_{2}\right) \\
& \left(k^{\sigma_{2}} M(-1+s)-k M^{\sigma_{2}} s \sigma_{2}+k M^{\sigma_{2}} s \sigma_{2}^{2}\right)+k M^{\sigma_{2}} s C(z)^{\sigma_{1}}\left(-1+\sigma_{1}\right)
\end{aligned}
$$




$$
\begin{aligned}
& \left.\left.\left.\left(-1+\sigma_{2}\right)\left(k^{\sigma_{2}} M(-1+s)-k M^{\sigma_{2}} s \sigma_{2}+k M^{\sigma_{2}} s \sigma_{2}^{2}\right)\right)\right)\right) / \\
& \left.\left(k^{\sigma_{2}} M(-1+s)-k M^{\sigma_{2}} s+k M^{\sigma_{2}} s \sigma_{2}\right)^{2}\right)
\end{aligned}
$$

and the trace is

$$
\begin{aligned}
& \operatorname{Tr}(\mathscr{J}) \\
& =1-\frac{b k^{1+\beta} \bar{m}^{\beta}}{\bar{M}^{2}}+\left(k ^ { - \beta } \overline { m } ^ { - 1 - \alpha } \overline { M } ^ { - \sigma _ { 2 } } \left(b k^{1+\alpha+\sigma_{2}} \bar{m}^{\beta} \bar{M}^{\sigma_{2}}(-1+s) \beta C\left(-1+\sigma_{2}\right)\right.\right. \\
& \quad+b k^{1+\alpha+\sigma_{2}} \bar{m}^{\beta} \bar{M}^{\sigma_{2}} s \beta C^{\sigma_{1}}\left(-1+\sigma_{1}\right)\left(-1+\sigma_{2}\right)+\left(k^{\beta} \bar{m}^{1+\alpha} \bar{M}^{\sigma_{2}} \pi \alpha\right. \\
& \left.\left.\left.\quad+b k^{\alpha+\sigma_{2}} \bar{m}^{\beta} w \beta\right)\left(-1+\sigma_{1}\right)\left(k^{\sigma_{2}} M(-1+s)-k \bar{M}^{\sigma_{2}} s+k \bar{M}^{\sigma_{2}} s \sigma_{2}\right)\right)\right) / \\
& \quad \times\left(\pi \alpha\left(-1+\sigma_{1}\right)\left(k^{\sigma_{2}} \bar{M}(-1+s) \quad-k M^{\sigma_{2}} s+k \bar{M}^{\sigma_{2}} s \sigma_{2}\right)\right)
\end{aligned}
$$

One can see that studying analytically the stability properties of this case (convex combination of preferences) is simply unbearable given the expression of the determinant.

\section{Appendix 3: Examples of alternative preferences}

In order to illustrate various aspects of the general results, we provide two examples of alternative utility functions: the logarithmic and the quadratic. The former is well known to be a special case of the CRRA utility function.

\section{The logarithmic utility}

We use a simple logarithm form for the utility functions of consumption and health. The other functions remain the same: $U(C)=\ln (C), \varphi(M)=\ln (M), \psi(m)=b^{\beta}$ and $h(m)=\pi m^{\alpha}$. We assume that the preferences are additive. The dynamic system of the economy becomes:

$$
\begin{aligned}
& \frac{\dot{\hat{C}}(z)}{\hat{\hat{C}}(z)}=r(z)-\rho \\
& \frac{\dot{\hat{m}}(z)}{\hat{\hat{m}}(z)}=\frac{-b \beta(w+C(z)) m(z)^{\beta-\alpha}}{\pi \alpha(\alpha-\beta) M(z)}+\frac{\delta_{M}+r(z)}{\alpha-\beta} \\
& \dot{\hat{M}}(z)=-\delta_{M}+\frac{b m^{\beta}}{M(z)} \\
& \dot{\hat{k}}(z)=B(M(z)) \hat{k}(z)^{\epsilon}-\hat{C}(z)-\hat{m}(z)-\delta \hat{k}(z)
\end{aligned}
$$

As we can see, there is a unique root, say $f(M)$ for the system (55)-(58). To obtain $f(M)$, we set (56)-(58) to zero for $k=\frac{\epsilon}{(1-\epsilon)(r+\delta)} w$. Then, replacing $m$ by its expression from (57) leads to: 

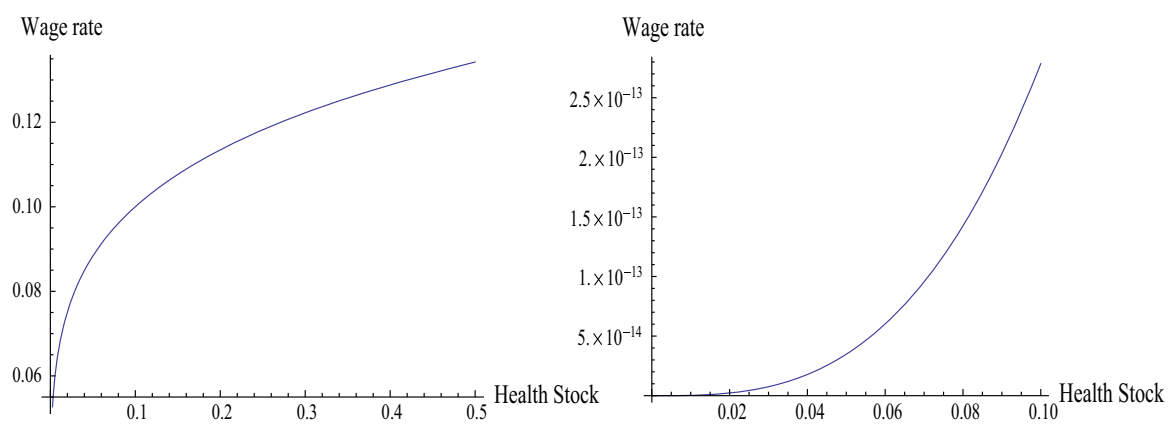

Fig. 12 The logarithmic utility: relation between wage rate and health stock for parameter values

$$
f(M)=M^{\frac{\alpha}{\beta}}-\frac{\delta_{M} \beta\left(\frac{\delta_{M}}{b}\right)^{-\frac{\alpha}{\beta}}}{\pi\left(\delta_{M}+r\right) \alpha}\left[\frac{2\left(r+\delta_{M}\right)-\delta \epsilon}{(1-\epsilon)(r+\delta)} w-\left(\frac{\delta_{M}}{b}\right)^{\frac{1}{\beta}}\right] M^{\frac{1}{\beta}}
$$

The unique solution follows from the fact that when we set $f(M)=0$, then the first component increases with $M$ and the second decreases. We now show how the wage rate depends on health. For that, we express $w$ as a function of $M$ by solving $f(M)=0$ :

$$
w(M)=\frac{(r+\delta)(1-\epsilon)}{\delta_{M} \beta(2 r+2 \delta-\delta \epsilon)}\left(\frac{\delta_{M}}{b}\right)^{\frac{1}{\beta}}\left[\left(\frac{\delta_{M}}{b}\right)^{\alpha}\left(\delta_{M}+r\right) \pi \alpha M^{\frac{\alpha}{\beta}}+\delta_{M} \beta M^{\frac{1}{\beta}}\right]
$$

The wage rate is an increasing function of the stock of health. Its evolution depends on the relative values of the degrees of homogeneity of the health production and the cost of investment function (i.e., on $\alpha$ and $\beta$ ). This is shown in a simulation exercise as plotted in Fig. 12. The left-hand side figure displays the relation for parameter values $\left\{\alpha, \beta, \delta_{M}, r, b, \epsilon, \delta, \pi\right\}=\{0.2,5,0.01,0.02,1,0.4,0.05,2\}$ and the right-hand side is for $\{4,0.2,0.01,0.02,1,0.4,0.05,2\}$. Albeit in both cases, the wage rate is an increasing function of the stock of health, the pattern of increase differs.

Indeed, when the growth rate of investment in health is lower than the one of its cost, meaning when $\beta>\alpha$, the stock of health increases faster than the wage rate, up to a certain level, say $M_{0}$, which is an inflexion point after which the relative evolution of the two variables changes ( $w$ growing faster than $M$ ). To obtain this inflexion point, we observe that $w(M)$ is of the form $a M^{\frac{\alpha}{\beta}}+b M^{\frac{1}{\beta}}$. Setting the second derivative of that expression to zero yields:

$$
M_{0}=\left[\frac{\beta-1}{\pi \alpha(\alpha-\beta)\left(\delta_{M}+r\right)}\left(\frac{\delta_{M}}{b}\right)^{-\alpha}\right]^{\frac{\beta}{\alpha-1}}
$$


In the case where $\beta<\alpha$, there is no inflexion point at which the curve becomes concave. To complete the example, let us briefly study the equilibrium dynamics. In that case, the Jacobian matrix turns out to be:

$$
\mathscr{J}=\left(\begin{array}{ccc}
1 & 1 & 0 \\
1-\frac{b k^{\alpha-\beta} m^{-\alpha+\beta} \beta}{M \pi \alpha} & 1-\frac{b k^{1+\alpha-\beta} m^{-1-\alpha+\beta}\left(\frac{c}{k}+w\right) \beta(-\alpha+\beta)}{M \pi \alpha} & \frac{b k^{1+\alpha-\beta} m^{-\alpha+\beta}\left(\frac{c}{k}+w\right) \beta}{M^{2} \pi \alpha} \\
1 & 1+\frac{b k^{1-\beta} m^{-1+\beta} \beta}{M} & -\frac{b k^{1-\beta} m^{\beta}}{M^{2}}
\end{array}\right)
$$

The determinant of $\mathscr{J}$ is computed as:

$$
\begin{aligned}
& \operatorname{det}(\mathscr{J})=-\frac{\beta b^{2} k^{1+\alpha-2 \beta} m^{-1-\alpha+2 \beta}(m+(c+k+w) \alpha)}{M^{3} \pi \alpha(\alpha-\beta)} \\
& \operatorname{Tr}(\mathscr{J})=2-\frac{b k^{1-\beta} m^{\beta}}{M^{2}}+\frac{b k^{\alpha-\beta} m^{-1-\alpha+\beta}(c+k w) \beta}{M \pi \alpha}
\end{aligned}
$$

One can easily check that $\operatorname{det}(\mathscr{J})<0$ if $\alpha>\beta$. This leads to the conclusion that the system admits a saddle point if $\alpha>\beta$. One of the conclusions that we can draw from the specific logarithmic utility is that, although this specification is free of the elasticity of substitution parameter used in CRRA utility framework, the core findings hold.

\section{The quadratic utility}

Unlike the preceding example in which the logarithmic specification is a particular case of the CRRA, the quadratic function departs sufficiently from it. Here, we will illustrate the three cases of preferences: additive, multiplicative and the convex combination. We assume functions of the forms:

$$
\begin{aligned}
& U(C(z))=C(z)-\eta_{1}(C(z))^{2} \\
& \varphi(M(z))=M(z)-\eta_{2}(M(z))^{2}
\end{aligned}
$$

with $\eta_{1} \neq 0$ and $\eta_{2} \neq 0$. As before, the health functions $\psi$ and $h$ maintain the same functional form: $\psi(m(z))=b m^{\beta}$ and $h(m(z))=\pi m^{\alpha}$, and we also assume the same production function.

\section{Separable additive case}

In this setting, the equilibrium values are given by $\bar{c}^{*}=\frac{1}{2 \eta_{1}}, \bar{M}^{*}=\frac{1}{2 \eta_{2}}, \bar{m}^{*}=$ $\left(\frac{\delta_{M}}{b}\right)^{\frac{1}{\beta}} \frac{1}{\left(2 \eta_{1}\right)^{\frac{1}{\beta}}}, \bar{k}^{*}=\frac{\epsilon}{2(r+\delta-\delta \epsilon) \eta_{1}}\left[1+\eta_{1}\left(2 \eta_{2} \frac{b}{\delta_{M}}\right)^{-\frac{1}{\beta}}\right]$ and $w^{*}(M)=\frac{(r+\delta)(1-\epsilon)}{2(r+\delta-\delta \epsilon) \eta_{1}}$ $\left[1+\eta_{1}\left(2 \eta_{1} \frac{b}{\delta_{M}}\right)^{-\frac{1}{\beta}} M^{* \frac{1}{\beta}}\right]$. We see that the equilibrium value of wage rate $w^{*}$ is determined by the stock of health under the conditions of Proposition 2. 


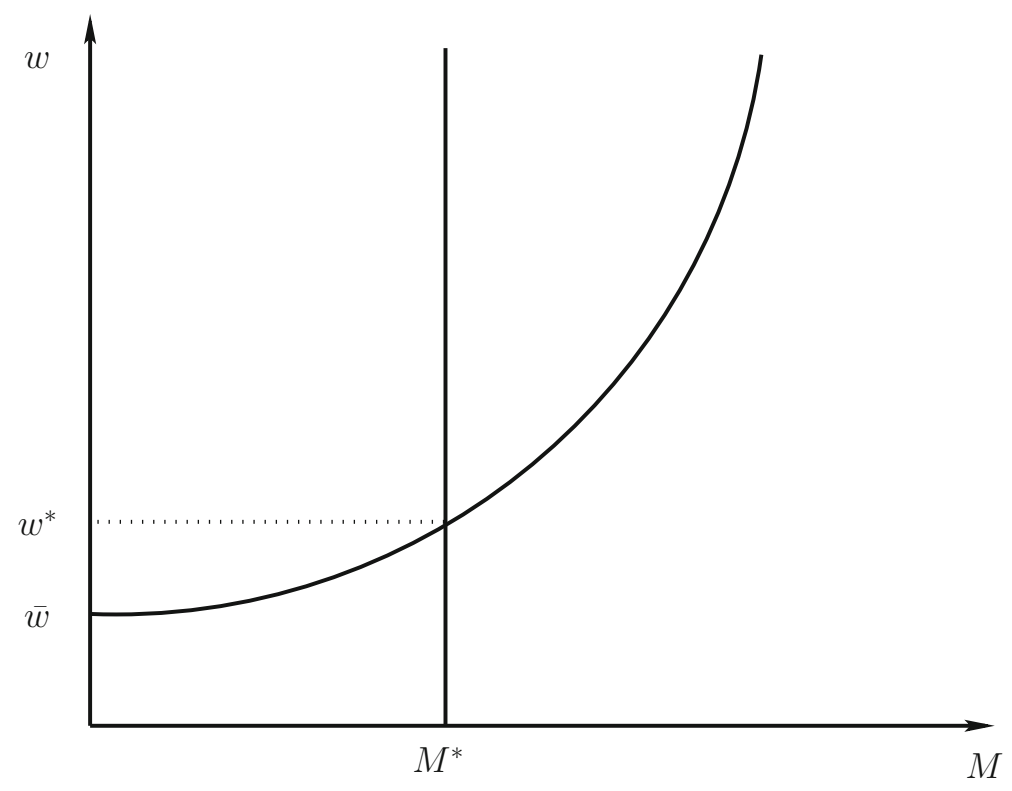

Fig. 13 The quadratic utility: evolution of equilibrium stock of health for additive preferences

Figure 13 shows the relationship between the wage rate and the stock of health. The vertical line represents the stock of health equilibrium $M^{*}$ and $w^{*}$ the wage rate at equilibrium; $\bar{w}=\frac{(r+\delta)(1-\epsilon)}{2(r+\delta-\delta \epsilon) \eta_{1}}$ denotes the intercept or the minimum wage. The wage rate in this example is an increasing function of the stock of health.

\section{Multiplicative case}

The equilibrium value of the stock of health is:

$$
\bar{M}^{*}(z)=\frac{1-\sqrt{1-4 \eta_{2} \mathrm{e}^{-(r-\rho) z}}}{2 \eta_{2}}
$$

Consumption at equilibrium is given by:

$$
\bar{c}^{*}=\frac{1}{2 \eta_{1}}+\frac{a_{0}}{a_{1}}+a_{2} w+\sqrt{\frac{1}{4 \eta_{1}^{2}}+\left(\frac{a_{0}}{a_{1}}+a_{2} w\right)^{2}}
$$

where $a_{0}=\pi\left(r+\delta_{M}\right) \eta_{1}\left(\frac{\delta_{M}}{b}\right)^{\frac{\alpha}{\beta}}\left(\bar{M}^{*}\right)^{\frac{\alpha}{\beta}}\left(\bar{M}^{*}-\eta_{2} \bar{M}^{* 2}\right), a_{1}=b \beta \frac{\delta_{M}}{b} \bar{M}^{*}\left(1-2 \eta_{2} \bar{M}^{*}\right)$ and $a_{2}=-\bar{M}^{*}+\eta_{2} \bar{M}^{* 2}$. The balanced wage rate $w^{*}(M)$ can be retrieved from Eq. (63). However, its expression is rather complex. From Eq. (62), one observes that $\bar{M}^{*}(z)$ depends on time $z$ and can be represented as in Fig. 14. 


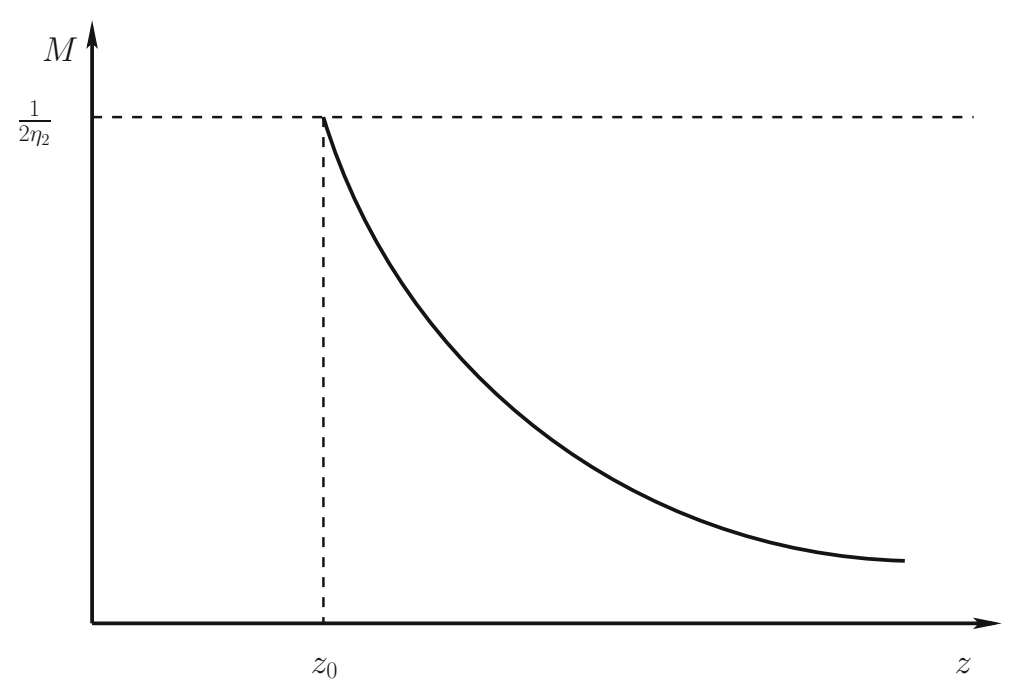

Fig. 14 The quadratic utility: evolution of equilibrium stock of health

The graph shows an initial time $z_{0}=\frac{\ln \left(4 \eta_{2}\right)}{r-\rho}$ under which there is no balance of stock of health. Moreover, the balanced path of the initial stock of health $\left(\frac{1}{2 \eta_{2}}\right)$ in the multiplicative model corresponds to the balanced path of the stock of health in the additive model, the latter being represented by the horizontal line. In other words, there exists a maximum level $\left(\frac{1}{2 \eta_{2}}\right)$ that the stock of health cannot exceed. The stock of health in the multiplicative model decreases over time to the minimum value of zero. One conclusion we can draw from this example is that the multiplicative model is more general as it provides information on the steady state of the additive model.

\section{Convex combination}

The equilibrium value of the stock of health is given by:

$$
\bar{M}^{*}(z)=\frac{1}{2 \eta_{2}}-\frac{\sqrt{(1-s)(r-\rho)+4 s(r-\rho) \eta_{2} \kappa(s) \mathrm{e}^{-(r-\rho) z}}}{2 \eta_{2} \sqrt{(1-s)(r-\rho)}}
$$

where $\kappa(s) \propto-4 \mathrm{e}^{(1-s)(r-\rho) \eta_{2}}$. The equilibrium value is closely linked to parameter $s$, and it can be shown that $\bar{M}^{*}(z)$ increases with $s$. The longevity $T$ is computed as:

$$
T=T_{0}-\frac{1}{r-\rho} \ln \left(s+M_{\min }(1-s)-\eta_{2} M_{\min }^{2}(1-s)\right)
$$

where $T_{0}=\frac{1}{r-\rho} \ln \left(\frac{\kappa(s)}{4(r-\rho)}\right)$. 


\section{References}

Acemoglu, D., Johnson, S.: Disease and development: a reply to Bloom, Canning, and Fink. J. Polit. Econ. (2014) 122, 1367-1375 (2014)

Acemoglu, D., Johnson, S.: Disease and development: the effect of life expectancy on economic growth. J. Polit. Econ. 115, 925-985 (2007)

Ashraf, Q.H., Lester, A., Weil, D.: When does improving health raise GDP? In: Acemoglu, Daron, Rogoff, Kenneth, Woodford, Michael (eds.) NBER Macroeconomics Annual 2008, pp. 157-204. University of Chicago Press, Chicago (2008)

Blackaby, D.H., Latreille, P., Murphy, P.D., O'Leary, N.C., Sloane, P.J.: An analysis of reservation wages for the economically inactive. Econ. Lett. 97, 1-5 (2007)

Bloom, D.E., Canning, D., Fink, G.: Disease and development revisited. J. Polit. Econ. (2014) 122, 13551366 (2014)

Bloom, D., Mahal, A.: AIDS, flu and the black death: impacts on economic growth and well being. In: Bloom, D., Godwin, P. (eds.) The Economics of HIV and AIDS: The Case of South and South East Asia, pp. 22-52. Clarendon Press, Oxford (1997a)

Bloom, D., Mahal, A.: Does AIDS epidemic threaten economic growth. J. Econom. 77, 105-124 (1997b)

Boucekkine, R., Laffargue, J.P.: On the distributional consequences of epidemics. J. Econ. Dyn. Control 34, 231-245 (2010)

Brown, S., Roberts, J., Taylor, K.: Reservation wages, labour market participation and health. J. R. Stat. Soc. Ser. A 173, 501-529 (2010)

Cai, L.: Effects of health on wages of Australian men. Econ. Rec. 85, 290-306 (2009)

Chakraborty, S.: Endogenous lifetime and economic growth. J. Econ. Theory 116, 119-137 (2004)

Corrigan, P., Glomm, G., Mendez, F.: AIDS crisis and growth. J. Dev. Econ. 77, 107-124 (2005)

Cuddington, J.T., Hancok, J.D.: Assessing the impact of AIDS on the growth path of the Malawian economy. J. Dev. Econ. 43, 363-368 (1994)

Ehrlich, I., Chuma, H.: A model of the demand for longevity and the value of life extension. J. Polit. Econ. 98, 761-782 (1990)

Friis, H., Michaelsen, K.F.: Micronutrients and HIV infection: a review. Eur. J. Clin. Nutr. 52, 157-163 (1998)

Goenka, A., Liu, L.: Infectious diseases and endogenous fluctuations. Econ. Theory 50, 125-149 (2012)

Goenka, A., Liu, L., Nguyen, M.-H.: Infectious diseases and economic growth. J. Math. Econ. 50, 34-53 (2014)

Gordon, R.H., Blinder, A.S.: Market wages, reservation wages, and retirement decisions. J. Public Econ. 14, 277-308 (1980)

Grossman, M.: On the concept of health capital and demand for health. J. Polit. Econ. 80, 223-255 (1972)

Grossman, M., Benham, L.: Health, hours and wages. In: Perlman, M. (ed.) The Economics of Health and Medical Care, pp. 205-233. Macmillan, London (1974)

Hall, R., Jones, C.I.: The value of life and the rise in health spending. Q. J. Econ. 122, 39-72 (2007)

Haveman, R., Wolfe, B., Kreider, B., Stone, M.: Market work, wages, and men's health. J. Health Econ. 13, 163-182 (1994)

Kirkwood, T.B.L.: A systematic look at an old problem. Nature 451, 644-657 (2008)

Lancaster, T., Chesher, A.: An econometric analysis of reservation wages. Econometrica 51, 1661-76(1983)

Lee, L.: Health and wages: a simultaneous equation model with multiple discrete indicators. Int. Econ. Rev. 23, 199-221 (1982)

McDonald, S., Roberts, J.: AIDS and economic growth: a human capital approach. J. Dev. Econ. 80, 228-250 (2006)

Strittmater, A., Sunde, U.: Health and economic development-evidence from the introduction of public health care. J. Popul. Econ. 26, 1549-1584 (2013)

Weil, D.N.: Health and economic growth. In: Aghion, Durlauf, (eds.) The Handbook of Economic Growth, vol. 2B. North Holland, Amsterdam (2014)

Weil, D.N.: Accounting for the effect of health on economic growth. Q. J. Econ. 122, 1265-1306 (2007) 